\title{
ARBUSCULAR MYCORRHIZAL AND DARK SEPTATE ENDOPHYTE FUNGAL ASSOCIATIONS IN PLANTS OF DIFFERENT VEGETATION TYPES IN VELLIANGIRI HILLS OF WESTERN GHATS, SOUTHERN INDIA
}

\author{
T. Muthukumar ${ }^{1 *}$, K. Sathiyadash $^{1,2}$ and V. Valarmathi ${ }^{1}$ \\ ${ }^{1}$ Root and Soil Biology Laboratory, Department of Botany, Bharathiar University \\ Coimbatore 641 046, Tamil Nadu, India; E-mail: *tmkum@yahoo.com \\ ${ }^{2}$ Department of Botany, Thiagarajar College, Madurai 625 009, Tamil Nadu, India
}

(Received 21 June, 2017; Accepted 16 August, 2017)

In recent years more attention is being paid to the presence of various non-pathogenic root fungal associations in plants of natural ecosystems for their role in various ecosystem processes. Despite their widespread reports in various ecosystems worldwide, our knowledge on root endophyte fungal association in plants from natural vegetation is far from complete. We assessed the arbuscular mycorrhizal (AM) and dark septate endophyte (DSE) fungal association in plants of Velliangiri Hills of the southern Western Ghats region, due to limited information on the root fungal association in this region. Of the 147 plant taxa (belonging to 46 families) investigated from five different vegetation types ranging from montane grasslands to tropical rainforest, 141 were colonised by AM fungi and co-occurrence of DSE fungi along with AM fungi was observed in 74 plant taxa. We report AM and DSE fungal associations for the first time in 61 and 42 plant species, respectively. Determination of AM morphological types indicated the frequent occurrence of intermediate type and AM morphology is reported for the first time in 64 plant taxa. Spore morphotypes belonging to eleven species (in six genera) were isolated from the different vegetation types. Arbuscular mycorrhizal fungal spore numbers neither differed significantly among vegetation types nor were related to AM fungal colonisation. Spores of Funneliformis geosporum was the most frequent spore morphotypes. Dark septate endophyte fungal association occurred in plants of all the vegetation types and was most frequent in herbs. Though no significant relationship was found between AM and DSE fungal colonisation within roots, a positive association was found in the occurrence of these two fungal groups.

Key words: AM fungi, Arum-type, DSE fungi, Glomus, intermediate type, Paris-type

\section{INTRODUCTION}

Arbuscular mycorrhizal (AM) fungi are the most common and widespread soil fungi associating with plant roots. Belonging to the phylum Glomeromycota, these fungi are presumed to associate with around $86 \%$ of the vascular plant taxa (Brundrett 2009). The AM fungal hyphal network in the soil plays an important role not only in initiating root colonisation, but also mediate nutrient uptake and their distribution among plants (Smith and Read 2008). 
Thus, AM fungi create functional linkages between biotic and abiotic components of the soil system (Berruti et al. 2015), affecting ecosystem processes like plant growth and productivity, as well as the structure and diversity of natural plant communities (Yang et al. 2016). In spite of over a century of assessment of the mycorrhizal status of plant species from different ecosystems worldwide, only less than $10 \%$ of the vascular plants have been actually examined for their mycorrhizal status (Brundrett 2009). Even for the plant taxa whose mycorrhizal status is known, the data at times are inconsistent or conflicting due to various levels of misdiagnosis (Brundrett 2009). In addition, it is also essential to examine plant taxa over a range of environmental conditions and habitat types to ascertain factors that may possibly influence their mycorrhizal status.

Root colonisation patterns of AM fungi within plant roots are categorised as Arum-, Paris- or intermediate types based on the spread and location of the intraradical structures of the AM fungi (Dickson 2004). The colonisation pattern is termed Arum-type when the fungus spreads intercellularly with intracellular hyphae differentiating into arbuscules in root cortical cells. In the Paris-type AM morphology, the spread of the fungus is chiefly intracellular forming hyphal or arbusculate coils. The colonisation patterns sharing features of both Arum- and Paris-types are termed as intermediate types. Although root colonisation patterns have been reported for plants from different vegetation formations, these represent only a small percentage of plant taxa where mycorrhizal status have been investigated (Dickson et al. 2007). It has been suggested that Arum-type colonisation patterns predominate roots of plants with high root growth rates, while Paris-type colonisation patterns are formed by plants with slow growing and long lived roots (Brundrett et al. 1990, Dickson et al. 2007). Experimental evidence has shown substantial transport of phosphate to the plant host through the fungal interphase in the different types of AM morphologies (Dickson et al. 2007).

Arbuscular mycorrhizal fungal colonisation in plant roots are influenced by both the presence and density of AM fungal propagules. Even though, AM fungi can perennate through different types of propagules like soil hyphae and mycorrhizal roots, the major propagules of AM fungi are the spores (Berruti et al. 2015, Grilli et al. 2015). Spores are also used to determine AM fungal community composition in different ecosystems (Grilli et al. 2015). Only limited studies have examined the AM fungal community composition in tropical ecosystems (Öpik and Davison 2016). Such assessments included tropical vegetation in China (Yang et al. 2013, Yu et al. 2017, Zhang et al. 2004, Zhao et al. 2003), Panama (Mangan et al. 2004, Schappe et al. 2017), Brazil (da Silva et al. 2017, Sturmer and Siqueira 2006, Zangaro et al. 2007), Costa Rica (Lovelock et al. 2003, Picone 2000) and India (Devi et al. 2017, D'Souza and Rodrigues 2013, Kumar and Adholeya 2016, Muthukumar and Udaiyan 2000a, 2002, Muthukumar et al. 2006, Radhika and Rodrigues 2010, Ragupathy and Mahadevan 1993). 
Plant roots in natural habitats are also frequently colonised by fungi with dematiaceous, septate hyphae forming microsclerotia or moniliform hyphae. These fungi are denoted as dark septate endophyte (DSE) fungi and often coexist with different mycorrhizal fungi (Mandyam and Jumpponen 2005, Newsham 2011). Although previous studies indicate a negative, neutral or positive influence of DSE fungi on plant performance, recent studies do indicate a positive effect of these fungi on plant growth and yield (Zhang et al. 2012). Mandyam and Jumpponen (2005) concluded that DSE fungi are prevalent in various habitats, but information on their prevalence in tropical ecosystems is scanty. Only limited studies (e.g. Muthukumar and Prabha 2013, Muthukumar et al. 2006, Rains et al. 2003, Uma et al. 2010) have examined the occurrence of DSE fungal association in tropical plant species.

Velliangiri Hills (long. $6^{\circ} 40^{\prime}$ to $7^{\circ} 10^{\prime} \mathrm{E}$ and lat. $10^{\circ} 55^{\prime}$ to $11^{\circ} 10^{\prime} \mathrm{N}$ ) is a mountain range in the Nilgiri Biosphere Reserve of Western Ghats, Southern India. The mountain peaks vary in their altitudes from 520 to $1,800 \mathrm{~m}$ a. s. 1 . The mean annual rainfall is quite variable ranging from 500 to $7,000 \mathrm{~mm}$. The average temperature ranges between $0{ }^{\circ} \mathrm{C}$ during winter and $41{ }^{\circ} \mathrm{C}$ during summer. The variability in altitudes and climatic conditions has resulted in different vegetation types ranging from grassland to tropical rainforest covering these hill ranges supporting a wide variety of flora and fauna (Murugesan and Balasubramanian 2008). The $48 \mathrm{~km}^{2}$ hill range consists of around 1,715 species of angiosperms including 439 endemics (Murugesan 2005, see Ragupathy et al. 2008 and references there in). The vegetation in Velliangiri Hills is presently under immense threat as the result of various anthropogenic activities (Balasubramanian and Murugesan 2004, Ragupathy et al. 2008). A previous study has shown that AM and DSE fungal association occur in grasses of this region (Sathiyadash et al. 2010). Apart from that study, there is no information on root fungal associations in plants of Velliangiri Hills. So the aim of the present investigation was therefore to record the AM and DSE fungal association in plants of different vegetation types in Velliangiri Hills. We also assessed the extent of variation in fungal colonisation and AM fungal spore numbers across species, life-forms, and vegetation types. Further, we also isolated and identified spores of AM fungi in soil samples of the plant taxa investigated. The relationship between various fungal variables was also assessed.

\section{MATERIALS AND METHODS}

\section{Sampling}

The samples of roots and soils of 147 taxa (94 herbs, 19 shrubs, 15 under shrubs and 19 trees) were collected from five different vegetation types southern tropical thorn forest (ST), tropical moist deciduous forest (TM), tropical wet 
evergreen forest (TW), shola forest (SH) and southern montane humid grassland (SM) in June 2009 (Table 1). More details on the vegetation types have been provided by Murugesan (2005) and Murugesan and Balasubramaniam (2008). Three plants were sampled for each taxon. Herbs were usually dug out and roots for shrubs and trees were traced back to the stem. Roots were washed clear of soil and preserved in formalin, acetic acid and 70\% alcohol (5:5:90; $\mathrm{v} / \mathrm{v} / \mathrm{v}$ ) solution and transported to the laboratory for processing. Soil shaken from roots and adjacent to plant roots was collected; air dried and packed individually in polythene bags. One-half of these soil samples were used for the enumeration and isolation of AM fungal spores. The other half of the soil sample from all the plants of a vegetation type was bulked to form a composite soil sample. This composite soil sample was used for determining soil characters.

\section{Determination of soil characters}

The air dried composite soil samples were sieved ( $2 \mathrm{~mm}$ grid) and three subsamples of the composite sample were analysed for the following characters. Soil $\mathrm{pH}$ and EC was determined in 1:2.5, soil:water (v/v) solution after incubation for $20 \mathrm{~min}$, with digital $\mathrm{pH}$ and conductivity meters, respectively, soon after the soil samples were brought to the laboratory (Jones 2001). Total nitrogen $(\mathrm{N})$, was determined using the micro-Kjeldahl method, available phosphorus (P) was determined with the method by Bray and Kurtz (1945) and exchangeable potassium $(\mathrm{K})$ was measured with a digital flame photometer after extraction in an ammonium acetate solution (Jackson 1971).

Table 1

Soil characteristics (mean \pm standard error) and the number of plant taxa examined from the different vegetation types of Velliangiri Hills.

\begin{tabular}{lccccc}
\hline & \multicolumn{5}{c}{ Vegetation types $^{\#}$} \\
\cline { 2 - 6 } & ST & TM & TW & SH & SM \\
\hline Soil type & Sandy soil & Sandy soil & $\begin{array}{c}\text { Sandy } \\
\text { clay loam }\end{array}$ & $\begin{array}{c}\text { Sandy clay } \\
\text { soil }\end{array}$ & Clay soil \\
\hline $\mathrm{pH}$ & $7.40 \pm 0.09$ & $7.90 \pm 0.09$ & $7.10 \pm 0.10$ & $7.97 \pm 0.11$ & $7.42 \pm 0.07$ \\
Ec $\left(\mathrm{dSm}^{-1}\right)$ & $0.10 \pm 0.01$ & $0.60 \pm 0.02$ & $0.60 \pm 0.01$ & $0.69 \pm 0.22$ & $0.80 \pm 0.05$ \\
Nitrogen $(\mathrm{mg} / \mathrm{kg})$ & $7.60 \pm 0.24$ & $7.70 \pm 0.21$ & $7.30 \pm 0.13$ & $10.80 \pm 0.38$ & $16.20 \pm 0.36$ \\
Phosphorus $(\mathrm{mg} / \mathrm{kg})$ & $0.84 \pm 0.03$ & $0.62 \pm 0.02$ & $0.74 \pm 0.04$ & $0.13 \pm 0.01$ & $0.60 \pm 0.02$ \\
Potassium $(\mathrm{mg} / \mathrm{kg})$ & $24.00 \pm 1.41$ & $22.00 \pm 1.00$ & $23.00 \pm 0.84$ & $17.40 \pm 0.41$ & $16.20 \pm 0.36$ \\
No. of taxa examined & 34 & 39 & 42 & 06 & 26 \\
\hline
\end{tabular}

${ }^{\text {"ST }}$ = southern tropical thorn forest; TM = tropical moist deciduous forest; TW = tropical wet evergreen forest; $\mathrm{SH}$ = shola forest; $\mathrm{SM}$ = southern montane humid grassland 
Preparation of roots for AM and DSE fungal assessment

The root samples were washed free of FAA, cut into 1-cm pieces, cleared in $2.5 \% \mathrm{KOH}$ (Koske and Gemma 1989), acidified with $5 \mathrm{~N} \mathrm{HCl}$ and stained with trypan blue $(0.05 \%$ in lacto glycerol) overnight. Roots that remained dark after clearing were bleached using alkaline $\mathrm{H}_{2} \mathrm{O}_{2}$ prior to acidification. Ten to thirty stained root bits were examined for each plant with a compound microscope $(\times 400)$ for the presence of AM and DSE fungal structures. The percentage root length colonised by AM and DSE fungi and the root length containing different fungal structures were estimated according to the magnified intersection method (McGonigle et al. 1990). Ten intersection points were scored for each root bit. Only root specimens containing arbuscules/arbusculate coils were considered to be mycorrhizal. Melanised hyphae with regular septation and microsclerotia were used to designate DSE fungal association. The AM and DSE fungal status of plant species in the present study were compared to previous literature (Harley and Harley 1987, Wang and Qiu 2006) to assess the information on their endorhizal status.

\section{Determination of AM fungal morphology}

The AM-morphology was classified as Arum-, Paris- or intermediate types based on whether the fungal hyphae were passing through the intercellular spaces or within cells as coils, respectively, following the descriptions of Dickson (2004). We did not distinguish the intermediate subtype morphologies described by Dickson (2004) as only the whole and squashed roots were examined. However, when the parallel running hyphae were intracellular, the morphology was designated as intermediate type. The AM morphological types of the plant species was compared to those presented by Dickson et al. (2007).

\section{Isolation, enumeration, and identification of AM fungal spores}

Spores of AM fungi were recovered from $100 \mathrm{~g}$ soil samples by wet-sieving and decanting technique (Muthukumar et al. 1996). The residues from the sieves $(710$ and $37 \mu \mathrm{m})$ were collected over filter papers, spread on a Petri dish and viewed under a dissection microscope at $\times 40$ magnifications. Only intact spores were counted. For enumeration, spore clusters and sporocarps were considered as one unit. Permanent slides of AM fungal spores for identification were prepared by transferring all the intact AM fungal spores onto a glass slide containing polyvinyl-alcohol-lacto glycerol with or without Melzer's reagent (Schenck and Perez 1990). Spores were cracked open for observation of spore wall characters. Spore characters were compared with the original descriptions present in databases established by the Schüßler laboratory (http:// 
www.amf-phylogeny.com/) and cultural database of INVAM (http://www. invam.caf.wvu.edu). Arbuscular mycorrhizal fungal nomenclature is that of Schüßler and Walker (2010). The frequency of each AM fungal spore morphotype was calculated as the number of plant taxa in which spore of a particular morphotype occurred by the total number of plant taxa examined $\times 100$.

\section{Life-history attributes and plant nomenclature}

Each plant taxon sampled during the survey was categorised for its lifeform and life-cycle attributes as determined directly from the field observations or literature (Henry et al. 1987, 1989, Nair and Henry 1983, Prain 1981a, b, Toby and Hodd 1982). Plant nomenclature and authorities are as The Plant List (2013).

\section{Statistical analysis}

The data on soil characteristics were subjected to Analysis of Variance (ANOVA) and the means were separated using Duncan's Multiple Range Test (DMRT). Percentage frequency of occurrence of different fungal association in different habit was calculated using the formula: Number of taxa in a habit with specific fungal association/total number of taxa in the habit examined $\times 100$. Data on fungal colonisation and AM fungal spore numbers were analysed using Kruskal-Wallis non-parametric analysis to assess the significance of the variation among plant species and vegetation types. The relationship between various AM and DSE fungal variables were analysed by Pearson's correlation. We also used the probabilistic co-occurrence model using $\mathrm{R}$ package co-occur available on Comprehensive $\mathrm{R}$ Archive Network (https://CRAN.R-project.org/package=cooccur) to assess the nature of association (positive, negative or random) between AM and DSE fungi as well as the AM fungal species associated with different plant species (Griffith et al. 2016).

\section{RESULTS}

\section{Soil properties}

Soils of different vegetation types in Velliangiri Hills were sandy to sandy clay loam. All soils were near neutral to slightly alkaline and the $\mathrm{pH}$ significantly varied among the vegetation types $\left(\mathrm{F}_{4,24}=15.425 ; \mathrm{P}<0.001\right)$. The EC significantly differed among the vegetation types $\left(\mathrm{F}_{4,24}=6.866 ; \mathrm{P}<0.001\right)$. and ranged between $0.10 \mathrm{dSm}^{-1}$ and $0.80 \mathrm{dSm}^{-1}$. The $\mathrm{N}\left(\mathrm{F}_{4,24}=182.069\right), \mathrm{P}\left(\mathrm{F}_{4,24}=\right.$ 109.062) and $\mathrm{K}\left(\mathrm{F}_{4,24}=15.276\right)$ contents of the soils significantly $(\mathrm{P}<0.001)$ varied among the vegetation types and ranged from 7.6 to $16.2 \mathrm{mg} / \mathrm{kg}$, 0.13 to 0.84 $\mathrm{mg} / \mathrm{kg}$, and 16.2 to $24 \mathrm{mg} / \mathrm{kg}$, respectively. 
Occurrence of AM and DSE fungi in various vegetation types

One hundred and forty-one of the 147 plant taxa (46 families) examined from the different vegetation types had AM fungal structures in their roots (Table 2). Plants roots belonging to non-mycorrhizal families like Com-

Table 2

Life history attributes, arbuscular mycorrhizal, dark septate endophytic fungal association and colonisation morphology in angiosperms of Velliangiri Hills. Abbreviation: Vegetation type $=$ VT, Habit $=$ Ht, Fungal association $=$ Fa, AM type $=$ At, Plant status $=$ Ps

\begin{tabular}{|c|c|c|c|c|c|}
\hline Family/Plant species & $\mathrm{VT}^{\mathrm{a}}$ & $\mathrm{Ht}^{\mathrm{b}}$ & $\mathrm{Fa}^{\mathrm{c}}$ & $\mathrm{At}^{\mathrm{d}}$ & $\mathrm{Ps}^{\mathrm{e}}$ \\
\hline \multicolumn{6}{|l|}{ Acanthaceae } \\
\hline Andrographis lineata Nees & $\mathrm{SM}$ & $\mathrm{H}$ & $\mathrm{AM}^{*}$ & $\mathrm{I}^{*}$ & \\
\hline Justicia beddomei (C. B. Clarke) Bennet & ST & $S$ & $\mathrm{AM}^{*}, \mathrm{DSE}^{*}$ & $\mathrm{I}^{*}$ & \\
\hline Justicia tranquebariensis L. & ST & US & $\mathrm{AM}^{*}, \mathrm{DSE}^{*}$ & $A^{*}$ & \\
\hline Nilgirianthus asper Santapau & $\mathrm{SH}$ & $S$ & $\mathrm{AM}^{*}, \mathrm{DSE}^{*}$ & $\mathrm{I}^{*}$ & \\
\hline Pleocaulus sessilis (Nees) Bremek. & $\mathrm{SH}$ & $\mathrm{H}$ & $\mathrm{AM}^{*}$ & $\mathrm{~A}^{*}$ & \\
\hline Rostellularia japonica (Thunb.) J. L. Ellis & TW & $\mathrm{H}$ & $\mathrm{AM}^{*}$ & $\mathrm{~A}^{*}$ & \\
\hline Rungia apiculata Bedd. & TW & US & $\mathrm{AM}^{*}, \mathrm{DSE}^{*}$ & $P^{*}$ & \\
\hline Rungia latior Nees & TW & $\mathrm{H}$ & $\mathrm{AM}^{*}$ & $\mathrm{I}^{*}$ & \\
\hline Strobilanthes asperrimus Nees & SM & S & $\mathrm{AM}^{*}, \mathrm{DSE}^{*}$ & $\mathrm{I}^{*}$ & \\
\hline Strobilanthes sp. & $\mathrm{SM}$ & $\mathrm{H}$ & AM, DSE & A & \\
\hline \multicolumn{6}{|l|}{ Agavaceae } \\
\hline Sansevieria roxburghiana Schult. et Schult. f. & ST & $S$ & AM, DSE & I & EI \\
\hline \multicolumn{6}{|l|}{ Anacardiaceae } \\
\hline Mangifera indica $\mathrm{L}$. & ST & $\mathrm{T}$ & AM, DSE & $\mathrm{P}$ & EI \\
\hline \multicolumn{6}{|l|}{ Annonaceae } \\
\hline Goniothalamus sp. & TW & $\mathrm{H}$ & AM & I & \\
\hline \multicolumn{6}{|l|}{ Araceae } \\
\hline Arisaema leschenaultii Blume Rumph. & $\mathrm{TW}$ & $\mathrm{H}$ & $\mathrm{AM}$ & A & \\
\hline Arisaema tortuosum (Wall.) Schott. & TW & $\mathrm{H}$ & $\mathrm{AM}$ & I & \\
\hline \multicolumn{6}{|l|}{ Asclepiadaceae } \\
\hline Cynanchum callialatum Buch.-Ham. ex Wight & TW & S & $\mathrm{AM}$ & I & \\
\hline \multicolumn{6}{|l|}{ Asteraceae } \\
\hline Acmella paniculata (Wall. ex DC.) R. K. Jansen & TW & $\mathrm{H}$ & $\mathrm{AM}, \mathrm{DSE}^{*}$ & A & EI \\
\hline Ageratum houstonianum Mill. & TW & $\mathrm{H}$ & $\mathrm{AM}^{*}, \mathrm{DSE}^{*}$ & $A^{*}$ & \\
\hline Anaphalis beddomei Hook. f. & TW & US & $\mathrm{AM}^{*}$ & $\mathrm{I}^{*}$ & \\
\hline Anaphalis brevifolia DC. & TW & $\mathrm{H}$ & $\mathrm{AM}^{*}, \mathrm{DSE}^{*}$ & $\mathrm{I}^{*}$ & \\
\hline Anaphalis busua (Buch.-Ham.) DC. & TW & $\mathrm{H}$ & $\mathrm{AM}^{*}, \mathrm{DSE}$ & $\mathrm{I}^{*}$ & \\
\hline
\end{tabular}


Table 2 (continued)

\begin{tabular}{|c|c|c|c|c|c|}
\hline Family/Plant species & $\mathrm{VT}^{\mathrm{a}}$ & $\mathrm{Ht}^{\mathrm{b}}$ & $\mathrm{Fa}^{\mathrm{c}}$ & $A t^{\mathrm{d}}$ & $\mathrm{Ps}^{\mathrm{e}}$ \\
\hline Anaphalis elliptica (DC.) DC. & TW & $\mathrm{H}$ & $\mathrm{AM}^{*}$ & $\mathrm{~A}^{*}$ & \\
\hline Anaphalis lawii (Hook. f.) Gamble. & TW & $\mathrm{H}$ & $\mathrm{AM}^{*}$ & $\mathrm{I}^{*}$ & \\
\hline Bidens biternata (Lour.) Murr. et Sherff. & $\mathrm{TM}$ & $\mathrm{H}$ & $\mathrm{AM}^{*}, \mathrm{DSE}^{*}$ & $\mathrm{~A}^{*}$ & \\
\hline Bidens pilosa $\mathrm{L}$. & $\mathrm{TM}$ & $\mathrm{H}$ & $\mathrm{AM}^{*}, \mathrm{DSE}^{*}$ & $\mathrm{I}^{*}$ & \\
\hline Bidens sp. & $\mathrm{TM}$ & $\mathrm{H}$ & AM, DSE & I & \\
\hline Chromolaena odorata (L.) R. M. King et H. Rob. & ST & $\mathrm{S}$ & $\mathrm{AM}^{*}$ & $\mathrm{~A}^{*}$ & \\
\hline Conyza japonica (Thunb.) Less. ex Less. & TW & $\mathrm{H}$ & $\mathrm{AM}^{*}, \mathrm{DSE}^{*}$ & $\mathrm{I}^{*}$ & \\
\hline Conyza leucantha (D. Don) Ludlow et P. H. Raven & $\mathrm{TM}$ & $\mathrm{H}$ & $\mathrm{AM}^{*}$ & $\mathrm{~A}^{*}$ & \\
\hline Emilia scabra DC. & $\mathrm{SH}$ & $\mathrm{H}$ & $\mathrm{AM}^{*}$ & $\mathrm{~A}^{*}$ & \\
\hline Emilia sp. & $\mathrm{SH}$ & $\mathrm{H}$ & $\mathrm{AM}$ & A & \\
\hline Erigeron aegypticus L. & $\mathrm{TM}$ & $\mathrm{H}$ & $\mathrm{AM}^{*}$ & $\mathrm{~A}^{*}$ & \\
\hline Erigeron bonariensis L. & $\mathrm{TM}$ & $\mathrm{H}$ & $\mathrm{AM}^{*}, \mathrm{DSE}^{*}$ & $I^{*}$ & \\
\hline Erigeron trilobus (Decne.) Boiss. & $\mathrm{TM}$ & $\mathrm{H}$ & $\mathrm{AM}^{*}, \mathrm{DSE}^{*}$ & $\mathrm{~A}^{*}$ & \\
\hline Helichrysum sp. & $\mathrm{SM}$ & $\mathrm{H}$ & AM, DSE & I & \\
\hline \multicolumn{6}{|l|}{ Balsaminaceae } \\
\hline Impatiens jerdoniae Wight & TW & $\mathrm{T}$ & NM & - & \\
\hline Impatiens minor (DC.) Bennet & TW & $\mathrm{H}$ & $\mathrm{AM}^{*}$ & $\mathrm{I}^{*}$ & \\
\hline \multicolumn{6}{|l|}{ Boraginaceae } \\
\hline Cynoglossum zeylanicum (Vahl) Brand & $\mathrm{TM}$ & $\mathrm{H}$ & $\mathrm{AM}^{*}, \mathrm{DSE}^{*}$ & $\mathrm{~A}^{*}$ & \\
\hline \multicolumn{6}{|l|}{ Caesalpinaceae } \\
\hline Cassia sp. & ST & US & $\mathrm{AM}$ & A & \\
\hline \multicolumn{6}{|l|}{ Capparaceae } \\
\hline Crataeva magna (Lour.) DC. & ST & $\mathrm{T}$ & $\mathrm{AM}, \mathrm{DSE}^{*}$ & $\mathrm{~A}^{*}$ & EI \\
\hline \multicolumn{6}{|l|}{ Cannabaceae } \\
\hline Cannabis sativa $\mathrm{L}$. & $\mathrm{SM}$ & $\mathrm{H}$ & $\mathrm{AM}^{*}$ & $\mathrm{I}^{*}$ & EI \\
\hline \multicolumn{6}{|l|}{ Commelinaceae } \\
\hline Commelina imberbis Ehrenb. ex Hassk. & $\mathrm{SM}$ & $\mathrm{H}$ & $\mathrm{AM}^{*}$ & $\mathrm{I}^{*}$ & \\
\hline Commelina sp. & $\mathrm{SM}$ & $\mathrm{H}$ & $\mathrm{AM}$ & A & \\
\hline \multicolumn{6}{|l|}{ Cruciferae } \\
\hline Brassica juncea (L.) Czern. & TW & $\mathrm{H}$ & NM & - & EI \\
\hline \multicolumn{6}{|l|}{ Cucurbitaceae } \\
\hline Cucumis prophaterum $\mathrm{L}$. & ST & $\mathrm{H}$ & $\mathrm{AM}^{*}, \mathrm{DSE}^{*}$ & $\mathrm{I}^{*}$ & \\
\hline Gynostemma pentaphyllum (Thunb.) Makino & $\mathrm{TM}$ & $\mathrm{H}$ & $\mathrm{AM}^{*}$ & $I^{*}$ & \\
\hline Gynura sp. & $\mathrm{TM}$ & $\mathrm{H}$ & AM, DSE & $\mathrm{I}$ & \\
\hline
\end{tabular}


Table 2 (continued)

\begin{tabular}{|c|c|c|c|c|c|}
\hline Family/Plant species & $\mathrm{VT}^{\mathrm{a}}$ & $\mathrm{Ht}^{\mathrm{b}}$ & $\mathrm{Fa}^{\mathrm{c}}$ & $A t^{d}$ & $\mathrm{PS}^{\mathrm{e}}$ \\
\hline Mukia leiosperma (Wight et Arn.) Wight & ST & $\mathrm{H}$ & $\mathrm{AM}^{*}, \mathrm{DSE}^{*}$ & $I^{*}$ & \\
\hline Zehneria maysorensis (Wight et Arn.) Arn. & TW & $\mathrm{H}$ & $\mathrm{AM}^{*}, \mathrm{DSE}^{*}$ & $A^{*}$ & RA \\
\hline Zehneria scabra Sond. & TW & $\mathrm{H}$ & NM & - & EI \\
\hline \multicolumn{6}{|l|}{ Cyperaceae } \\
\hline Cyperus cyperinus (Retz.) Suringar & SM & $\mathrm{H}$ & $\mathrm{AM}$ & I & \\
\hline Cyperus rotundus L. & $\mathrm{SM}$ & $\mathrm{H}$ & NM & - & EI \\
\hline Cyperus sp. & $\mathrm{SM}$ & $\mathrm{H}$ & $\mathrm{AM}$ & I & \\
\hline Fimbristylis falcata (Vahl) Kunth & $\mathrm{SM}$ & $\mathrm{H}$ & $\mathrm{AM}, \mathrm{DSE}^{*}$ & A & \\
\hline Fimbristylis ovata (Burm. f.) J. Kern & $\mathrm{SM}$ & $\mathrm{H}$ & AM, DSE* & I & \\
\hline Fimbristylis strigosa Govind. & $\mathrm{SM}$ & $\mathrm{H}$ & $\mathrm{AM}, \mathrm{DSE}^{*}$ & $\mathrm{I}^{*}$ & \\
\hline \multicolumn{6}{|l|}{ Dioscoreaceae } \\
\hline Dioscorea oppositifolia L. & ST & $\mathrm{H}$ & $\mathrm{AM}^{*}$ & $\mathrm{~A}^{*}$ & \\
\hline Dioscorea tomentosa J. Koenig ex Spreng. & ST & $\mathrm{H}$ & $\mathrm{AM}$ & I & \\
\hline \multicolumn{6}{|l|}{ Eriocaulaceae } \\
\hline Eriocaulon sp. & $\mathrm{SM}$ & $\mathrm{H}$ & AM, DSE & $\mathrm{A}$ & \\
\hline \multicolumn{6}{|l|}{ Euphorbiaceae } \\
\hline Acalypha racemosa Wall. ex Baill. & ST & US & $\mathrm{AM}^{*}, \mathrm{DSE}^{*}$ & $\mathrm{~A}^{*}$ & EI \\
\hline Breynia retusa (Dennst.) Alston. & $\mathrm{TM}$ & $\mathrm{H}$ & $\mathrm{AM}^{*}$ & $\mathrm{I}^{*}$ & EI \\
\hline Bridelia retusa (L.) A. Juss. & $\mathrm{TM}$ & $\mathrm{T}$ & $\mathrm{AM}$ & I & EI \\
\hline Bridelia stipularis (L.) Blume & $\mathrm{TM}$ & $S$ & AM, DSE & I & \\
\hline Euphorbia indica Lam. & $\mathrm{TM}$ & $\mathrm{H}$ & $\mathrm{AM}^{*}$ & $\mathrm{~A}^{*}$ & EI \\
\hline Euphorbia tortilis Rottler ex Ainslie & $\mathrm{TM}$ & $\mathrm{H}$ & $\mathrm{AM}^{*}$ & $I^{*}$ & \\
\hline Flueggea leucopyrus Willd. & $\mathrm{TM}$ & $S$ & $\mathrm{AM}$ & I & \\
\hline Glochidion heyneanum (Wight et Arn.) Wight. & TW & $\mathrm{T}$ & AM, DSE & I & \\
\hline Mallotus philippensis (Lam.) Müll. Arg. & $\mathrm{TM}$ & $\mathrm{T}$ & AM, DSE & I & EI \\
\hline Mallotus tetracoccus (Roxb.) Kurz & $\mathrm{TM}$ & $\mathrm{T}$ & AM, DSE & I & \\
\hline Phyllanthus emblica L. & $\mathrm{TM}$ & $\mathrm{T}$ & $\mathrm{AM}$ & $\mathrm{A}^{*}$ & \\
\hline Phyllanthus maderaspatensis $\mathrm{L}$. & ST & $\mathrm{H}$ & $\mathrm{AM}^{*}$ & $\mathrm{~A}^{*}$ & \\
\hline Phyllanthus missionis Hook. f. & ST & US & $\mathrm{AM}$ & $\mathrm{A}$ & \\
\hline Phyllanthus reticulatus Poir. & ST & $S$ & $\mathrm{AM}^{*}, \mathrm{DSE}^{*}$ & $\mathrm{~A}^{*}$ & \\
\hline Phyllanthus urinaria L. & $\mathrm{TM}$ & $\mathrm{H}$ & $\mathrm{AM}$ & I & \\
\hline \multicolumn{6}{|l|}{ Gentianaceae } \\
\hline Exacum sp. & $\mathrm{SM}$ & $\mathrm{H}$ & $\mathrm{AM}$ & $\mathrm{P}$ & \\
\hline Swertia sp. & SM & $\mathrm{H}$ & $\mathrm{AM}$ & $\mathrm{P}$ & \\
\hline
\end{tabular}


Table 2 (continued)

\begin{tabular}{|c|c|c|c|c|c|}
\hline Family/Plant species & $\mathrm{VT}^{\mathrm{a}}$ & $\mathrm{Ht}^{\mathrm{b}}$ & $\mathrm{Fa}^{\mathrm{c}}$ & $A t^{\mathrm{d}}$ & $\mathrm{Ps}^{\mathrm{e}}$ \\
\hline \multicolumn{6}{|l|}{ Haloragaceae } \\
\hline Laurembergia coccinea Kanitz & TW & $\mathrm{H}$ & $\mathrm{AM}^{*}$ & $\mathrm{I}^{*}$ & \\
\hline \multicolumn{6}{|l|}{ Juncaceae } \\
\hline Juncus prismatocarpus $\mathrm{R}$. Br. & $\mathrm{SM}$ & $\mathrm{H}$ & $\mathrm{AM}^{*}, \mathrm{DSE}^{*}$ & $I^{*}$ & \\
\hline \multicolumn{6}{|l|}{ Labiatae } \\
\hline Leucas marrubioides Desf. & TW & $\mathrm{H}$ & $\mathrm{AM}^{*}$ & $\mathrm{~A}^{*}$ & \\
\hline Leucas rosmarinifolia Benth. & TW & $\mathrm{S}$ & $\mathrm{AM}^{*}, \mathrm{DSE}^{*}$ & $I^{*}$ & \\
\hline Leucas sp. & TW & $\mathrm{S}$ & AM, DSE & A & \\
\hline Orthosiphon pallidus Royle ex Benth. & ST & US & $\mathrm{AM}^{*}$ & $\mathrm{I}^{*}$ & \\
\hline Orthosiphon rubicundus (D. Don) Benth. & ST & $\mathrm{H}$ & $\mathrm{AM}^{*}, \mathrm{DSE}^{*}$ & $\mathrm{I}^{*}$ & EI \\
\hline Orthosiphon sp. & ST & $\mathrm{H}$ & AM, DSE & A & \\
\hline Pogostemon sp. & $\mathrm{SH}$ & $\mathrm{H}$ & AM, DSE & I & \\
\hline Scutellaria sp. & TW & $\mathrm{H}$ & AM, DSE & $\mathrm{I}$ & \\
\hline \multicolumn{6}{|l|}{ Lauraceae } \\
\hline Neolitsea scrobiculata Gamble & TW & $\mathrm{T}$ & $\mathrm{AM}$ & I & \\
\hline \multicolumn{6}{|l|}{ Liliaceae } \\
\hline Asparagus racemosus Willd. & $\mathrm{TM}$ & $\mathrm{H}$ & $\mathrm{AM}$ & $\mathrm{A}$ & \\
\hline Urginea indica (Roxb.) Kunth. & $\mathrm{TM}$ & $\mathrm{H}$ & $\mathrm{AM}$ & $\mathrm{A}$ & \\
\hline \multicolumn{6}{|l|}{ Malvaceae } \\
\hline Urena lobata $\mathrm{L}$. & ST & US & $\mathrm{AM}^{*}$ & $\mathrm{I}^{*}$ & EI \\
\hline Urena sp. & ST & $\mathrm{H}$ & $\mathrm{AM}$ & A & \\
\hline \multicolumn{6}{|l|}{ Mimosaceae } \\
\hline Albizia sp. & $\mathrm{TM}$ & $\mathrm{T}$ & AM, DSE & I & \\
\hline Pithecellobium dulce (Roxb.) Benth. & $\mathrm{TM}$ & $\mathrm{T}$ & AM, DSE & I & \\
\hline \multicolumn{6}{|l|}{ Oleaceae } \\
\hline Jasminum ritchiei C. B. Clarke & $\mathrm{TM}$ & S & $\mathrm{AM}$ & A & \\
\hline \multicolumn{6}{|l|}{ Oxalidaceae } \\
\hline Biophytum longipedunculatum Govind. & TW & $\mathrm{H}$ & $\mathrm{AM}^{*}, \mathrm{DSE}^{*}$ & A & \\
\hline Biophytum sensitivum (L.) DC. & TW & $\mathrm{H}$ & AM, DSE* & A & \\
\hline Oxalis corniculata L. & TW & $\mathrm{H}$ & AM, DSE & I & \\
\hline \multicolumn{6}{|l|}{ Papilionaceae } \\
\hline Crotalaria clavata Wight et Arn. & ST & $\mathrm{S}$ & $\mathrm{AM}$ & I & \\
\hline Crotalaria sp. & ST & US & $\mathrm{AM}$ & I & \\
\hline Crotalaria verrucosa L. & ST & $\mathrm{H}$ & AM, DSE* & $\mathrm{A}^{*}$ & \\
\hline
\end{tabular}


Table 2 (continued)

\begin{tabular}{|c|c|c|c|c|c|}
\hline Family/Plant species & $\mathrm{VT}^{\mathrm{a}}$ & $\mathrm{Ht}^{\mathrm{b}}$ & $\mathrm{Fa}^{\mathrm{c}}$ & $A t^{\mathrm{d}}$ & $\mathrm{Ps}^{\mathrm{e}}$ \\
\hline Pterocarpus marsupium Roxb. & ST & $\mathrm{T}$ & $\mathrm{AM}$ & $\mathrm{I}$ & \\
\hline Vigna unguiculata (L.) Walp. & ST & $\mathrm{H}$ & AM, DSE* & $\mathrm{A}$ & EI \\
\hline \multicolumn{6}{|l|}{ Plantaginaceae } \\
\hline Plantago erosa Wall. & $\mathrm{TM}$ & $\mathrm{H}$ & AM, DSE* & $\mathrm{A}$ & \\
\hline Plantago sp. & $\mathrm{TM}$ & $\mathrm{H}$ & AM, DSE & $\mathrm{A}$ & \\
\hline \multicolumn{6}{|l|}{ Poaceae } \\
\hline Arundinella sp. & $\mathrm{SM}$ & $\mathrm{H}$ & $\mathrm{AM}$ & I & \\
\hline Brachiaria sp. & SM & $\mathrm{H}$ & AM, DSE & I & \\
\hline Cymbopogon citratus (DC.) Stapf & TW & $\mathrm{H}$ & AM, DSE & I & EI \\
\hline Cyrtococcum sp. & TW & $\mathrm{H}$ & $\mathrm{AM}$ & I & \\
\hline Eleusine indica (L.) Gaertn. & TW & $\mathrm{H}$ & $\mathrm{AM}$ & $\mathrm{P}$ & \\
\hline $\begin{array}{l}\text { Heteropogon contortus (L.) P Beauv. ex Roem. et } \\
\text { Schultes }\end{array}$ & TW & $\mathrm{H}$ & AM, DSE* & I & \\
\hline Ischaemum sp. & TW & $\mathrm{H}$ & AM, DSE & A & \\
\hline Jansenella griffithiana (C. Muell) Bor. & $\mathrm{SM}$ & $\mathrm{H}$ & $\mathrm{AM}^{*}, \mathrm{DSE}^{*}$ & $\mathrm{P}^{*}$ & \\
\hline Panicum notatum Retz. & SM & $\mathrm{H}$ & $\mathrm{AM}^{*}$ & $A^{*}$ & \\
\hline Panicum sparsicomum Nees ex Steud. & $\mathrm{SM}$ & $\mathrm{H}$ & $\mathrm{AM}^{*}, \mathrm{DSE}^{*}$ & $\mathrm{I}^{*}$ & RA \\
\hline Polytrias indica (Houtt.) Veldkamp. & TW & $\mathrm{H}$ & AM & I & \\
\hline Tripogan sp. & $\mathrm{SM}$ & $\mathrm{H}$ & $\mathrm{NM}$ & - & \\
\hline \multicolumn{6}{|l|}{ Polygalaceae } \\
\hline Polygala sp. & $\mathrm{SM}$ & $\mathrm{H}$ & AM, DSE & I & \\
\hline \multicolumn{6}{|l|}{ Polygonaceae } \\
\hline Persicaria barbata (L.) H. Hara & $\mathrm{SM}$ & $\mathrm{H}$ & $\mathrm{AM}^{*}$ & $\mathrm{I}^{*}$ & EI \\
\hline Persicaria chinense (L.) H. Gross. & TW & US & $\mathrm{AM}^{*}, \mathrm{DSE}^{*}$ & $\mathrm{I}^{*}$ & EI \\
\hline Persicaria lanigerum (R. Br.) Sojak. & TW & $\mathrm{H}$ & $\mathrm{AM}^{*}$ & A & \\
\hline \multicolumn{6}{|l|}{ Rhamnaceae } \\
\hline Ziziphus rugosa Lam. & $\mathrm{TM}$ & $\mathrm{T}$ & $\mathrm{AM}^{*}, \mathrm{DSE}^{*}$ & $I^{*}$ & EI \\
\hline \multicolumn{6}{|l|}{ Rosaceae } \\
\hline Rubus ellipticus Sm. & TW & $S$ & $\mathrm{AM}^{*}$ & $\mathrm{~A}^{*}$ & EI, B \\
\hline \multicolumn{6}{|l|}{ Rubiaceae } \\
\hline Canthium rheedi DC. & $\mathrm{SH}$ & $\mathrm{H}$ & $\mathrm{AM}^{*}$ & $A^{*}$ & \\
\hline Canthium travancoricum Bedd. & TW & $\mathrm{T}$ & $\mathrm{AM}$ & I & \\
\hline Catunaregam spinosa (Thunb.) Triveng. & TW & $\mathrm{T}$ & AM, DSE & I & \\
\hline Knoxia sumatrensis (Retz.) DC. & SM & $\mathrm{H}$ & $\mathrm{AM}$ & $\mathrm{I}$ & \\
\hline
\end{tabular}


Table 2 (continued)

\begin{tabular}{|c|c|c|c|c|c|}
\hline Family/Plant species & $\mathrm{VT}^{\mathrm{a}}$ & $\mathrm{Ht}^{\mathrm{b}}$ & $\mathrm{Fa}^{\mathrm{c}}$ & $A t^{d}$ & $\mathrm{Ps}^{\mathrm{e}}$ \\
\hline Psydrax dicoccos Gaertn. & TW & $\mathrm{T}$ & $\mathrm{AM}$ & $\mathrm{P}$ & RA \\
\hline \multicolumn{6}{|l|}{ Rutaceae } \\
\hline Clausena heptaphylla (Roxb.) Wight et Arn. & $\mathrm{TM}$ & $S$ & NM & - & \\
\hline Glycosmis sp. & $\mathrm{TM}$ & $S$ & $\mathrm{AM}$ & I & \\
\hline Toddalia asiatica (L.) Lam. & $\mathrm{TM}$ & $S$ & AM, DSE & A & \\
\hline \multicolumn{6}{|l|}{ Sapindaceae } \\
\hline Dimocarpus longan Lour. & $\mathrm{TM}$ & $\mathrm{T}$ & $\mathrm{AM}$ & A & EI \\
\hline Filicium decipiens (Wight et Arn.) Thwaites & $\mathrm{TM}$ & $\mathrm{T}$ & AM, DSE & I & EI \\
\hline \multicolumn{6}{|l|}{ Scrophulariaceae } \\
\hline Scoparia dulcis L. & $\mathrm{TM}$ & US & $\mathrm{AM}^{*}$ & $\mathrm{I}^{*}$ & \\
\hline Torenia hirsuta Willd. & $\mathrm{TM}$ & $\mathrm{H}$ & $\mathrm{AM}^{*}, \mathrm{DSE}^{*}$ & $\mathrm{I}^{*}$ & \\
\hline \multicolumn{6}{|l|}{ Solanaceae } \\
\hline Solanum americanum Mill. & ST & $\mathrm{H}$ & $\mathrm{AM}$ & $\mathrm{A}$ & EI \\
\hline Solanum ferox $\mathrm{L}$. & ST & $\mathrm{H}$ & $\mathrm{AM}^{*}, \mathrm{DSE}^{*}$ & $\mathrm{I}^{*}$ & \\
\hline Solanum grandiflorum Ruiz et Pav. & ST & US & $\mathrm{AM}^{*}$ & $I^{*}$ & \\
\hline Solanum trilobatum $\mathrm{L}$. & ST & US & $\mathrm{AM}^{*}, \mathrm{DSE}^{*}$ & $\mathrm{I}^{*}$ & EI \\
\hline Solanum viarum Dunal & ST & US & $\mathrm{AM}^{*}$ & $I^{*}$ & EI \\
\hline \multicolumn{6}{|l|}{ Thunbergiaceae } \\
\hline Thunbergia fragrans Roxb. & ST & $\mathrm{H}$ & AM, DSE* & I & \\
\hline Thunbergia sp. & ST & $\mathrm{H}$ & AM, DSE & I & \\
\hline \multicolumn{6}{|l|}{ Umbelliferae } \\
\hline Polyzygus tuberosus Walp. & $\mathrm{TM}$ & $\mathrm{H}$ & AM, DSE* & I & \\
\hline \multicolumn{6}{|l|}{ Urticaceae } \\
\hline Pouzolzia sp. & $\mathrm{TM}$ & US & AM, DSE & I & \\
\hline \multicolumn{6}{|l|}{ Verbenaceae } \\
\hline Lantana camara $\mathrm{L}$. & ST & $S$ & AM, DSE* & A & EI \\
\hline Tectona grandis L. & ST & $\mathrm{T}$ & AM, DSE & I & \\
\hline \multicolumn{6}{|l|}{ Vitaceae } \\
\hline Cayratia auriculata (Roxb.) Gamble & $\mathrm{TM}$ & $\mathrm{H}$ & AM, DSE & I & \\
\hline Tetrastigma sp. & $\mathrm{TM}$ & $S$ & AM, DSE & I & \\
\hline
\end{tabular}

aST = southern tropical thorn forest; $\mathrm{TM}=$ tropical moist deciduous forest; $\mathrm{TW}=$ tropical wet evergreen forest; $\mathrm{SH}=$ shola forest; $\mathrm{SM}=$ southern montane humid grassland; ${ }^{\mathrm{b}} \mathrm{H}=$ herb; $\mathrm{S}=$ shrub; US = under shrub; $\mathrm{T}=$ tree; ${ }^{\mathrm{c}} \mathrm{AM}=$ arbuscular mycorrhizal; DSE $=$ dark septate endophyte; $\mathrm{NM}=$ non-mycorrhizal; ${ }^{\mathrm{d}} \mathrm{A}=$ Arum-type; $\mathrm{P}=$ Paris-type; $\mathrm{I}=$ intermediate type; ${ }^{\mathrm{e}} \mathrm{EI}=$ economically important; $\mathrm{B}=$ species useful in plant breeding; $\mathrm{RA}=$ rare; ${ }^{*}=$ first report 


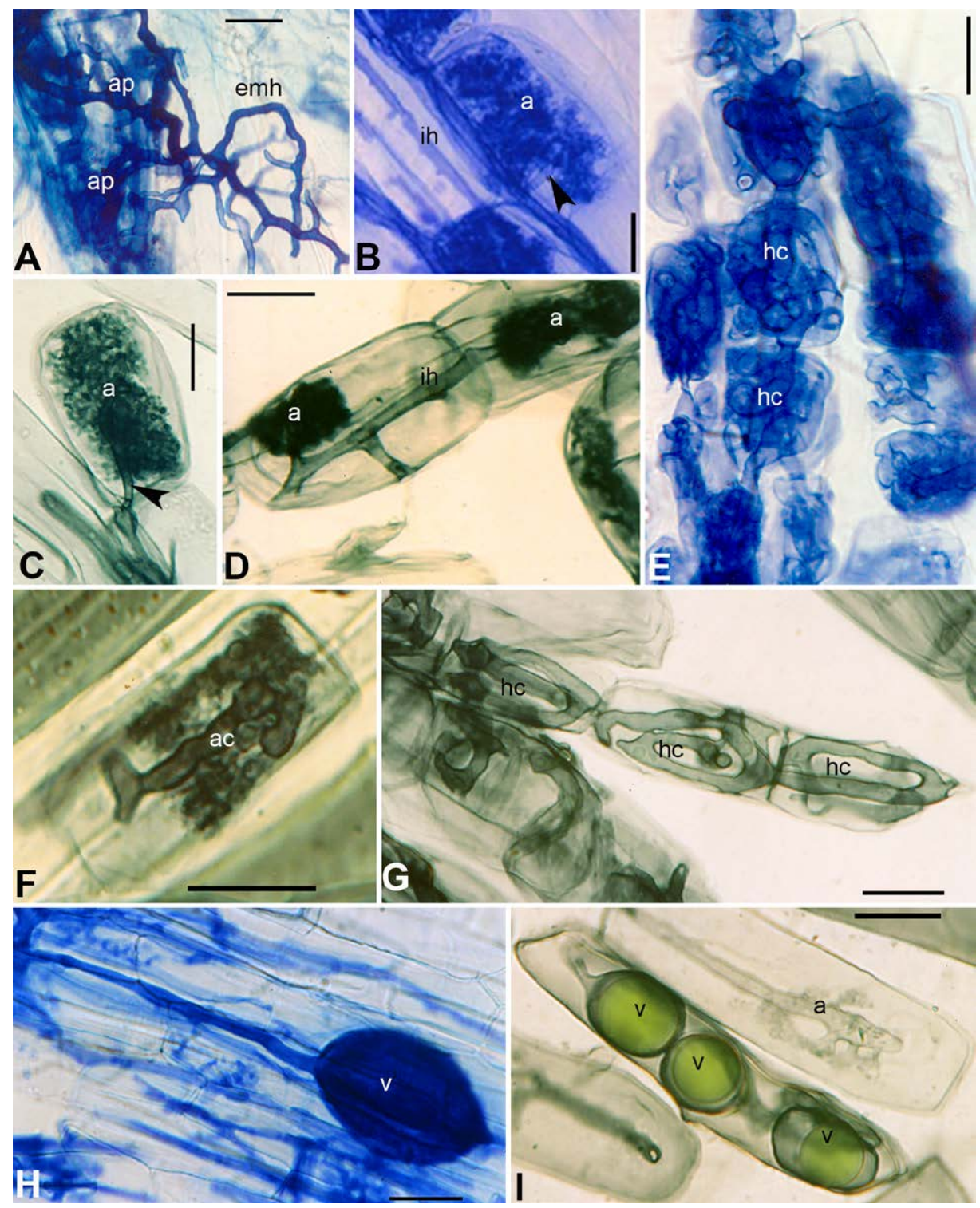

Fig. 1. Arbuscular mycorrhizal (AM) fungal association and morphology in plants of Velliangiri Hills. $\mathrm{A}=$ Extraradical hyphae $(\mathrm{emh})$ and appressoria $(\mathrm{ap})$ on the root surface of Scutellaria sp.; B = intracellular linear hyphae (ih), arbuscule (a) and arbuscular trunk (arrow head) in Phyllanthus emblica; $\mathrm{C}=$ arbuscular trunk (arrow head) and arbuscule (a) in Brachiaria sp.; D = intracellular linear hyphae (ih) and arbuscule (a) in Panicum notatum; E = intracellular hyphal coils (hc) in Swertia sp.; F = arbusculate coils (ac) in Polygala sp.; G = intracellular hyphal coils (hc) in Orthosiphon sp.; $\mathrm{H}=$ intercellular vesicle (v) in Nilgirianthus asper; I = intracellular vesicles (v) and arbuscule (a) in Polygala sp. Scale bars: $50 \mu \mathrm{m}$ 
melinaceae, Cyperaceae, Capparaceae and Juncaceae had AM fungal structures. The entry of AM fungi into roots was characterised by an appressorium on the root surface (Fig. 1A). The AM fungal structures within roots included arbuscules, intra- and intercellular hyphae, hyphal and arbusculate coils and inter or intracellular vesicles (Fig. 1B-C). Non-mycotrophy was low $(4 \%$, 6/147) and occurred in plant species of SM, TM, and TW. All the plant species examined from the ST were mycorrhizal. Ninety-two percent $(24 / 26)$ of the plant species in SM, 97\% (38/39) from the TM and 93\% (39/42) from TW were mycorrhizal (Fig. 3).

Melanised, regularly septate hyphae, and microsclerotia characterised DSE fungal associations (Fig. 2A-D) and were found in 74 plant roots examined from different vegetation types. DSE fungal association was more frequent $(62 \%)$ in southern tropical thorn forests compared to other forest types (Table 2 and Fig. 3). Plant species in six of nine monocot families and 26 of 37 dicot families had DSE fungal association. All the plants possessing DSE fungal association also harboured AM fungi. Further, $88 \%$ of the economically impor-

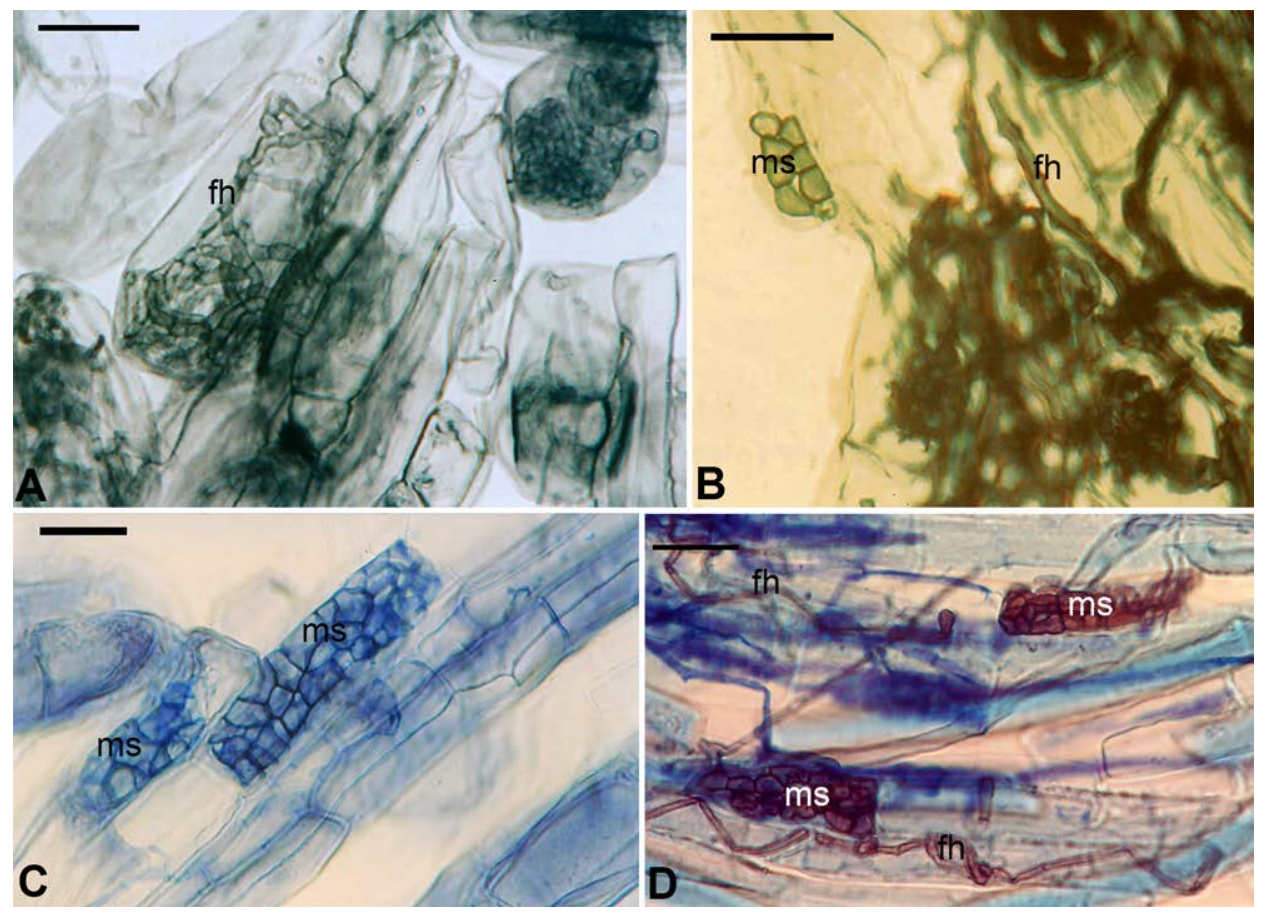

Fig. 2. Dark septate endophyte (DSE) fungal association in angiosperms of Velliangiri Hills. $\mathrm{A}=$ Dark septate intracellular fungal hyphae (fh) in Scutellaria sp.; B = septate fungal hyphae (fh) and microsclerotia (ms) in Torenia sp.; $\mathrm{C}=$ microsclerotia (ms) in Nilgirianthus asper; $\mathrm{D}=$ septate fungal hyphae (fh) and microsclerotia (ms) in Brachiaria sp. Scale bars: $50 \mu \mathrm{m}$ 
tant plant species had AM and 30\% had DSE fungal association. Of the three rare plant species examined in the present study, Zehneria maysorensis (Cucurbitaceae) and Panicum sparsicomum (Poaceae) had dual colonisation of AM and DSE and Psydrax dicoccos (Rubiaceae) was colonised only by AM fungi.

\section{$A M$ and DSE fungal association in plant life-forms in different vegetation types}

All the herbs examined from ST and TM were mycorrhizal. Ninety-two percent of the herbs in the SM and 93\% of herbs in the TW were mycorrhizal. All shrubs examined from the ST, TW, SM and $86 \%$ of those examined from the TM were colonised by AM fungi. All the under shrubs examined from three forests such as ST, TM, and TW had AM fungal associations. Similarly, all the trees in the ST, TM forests and $83 \%$ of tree species in the TW had AM fungal associations (Table 2 and Fig. 3).

Herbs were frequently colonised by DSE fungi in all the vegetation types. Thirty-eight percent of the herbs in the SM, $90 \%$ of herbs in the ST, 57\% in the TM and $46 \%$ in TW had DSE fungal associations. Thirty percent of the under shrubs in the ST and 50\% in the TM had DSE fungal association. Seventy-five percent of trees from the ST, $67 \%$ from the TM and $17 \%$ from the TW are colonised by AM fungi respectively (Table 2 and Fig. 3).

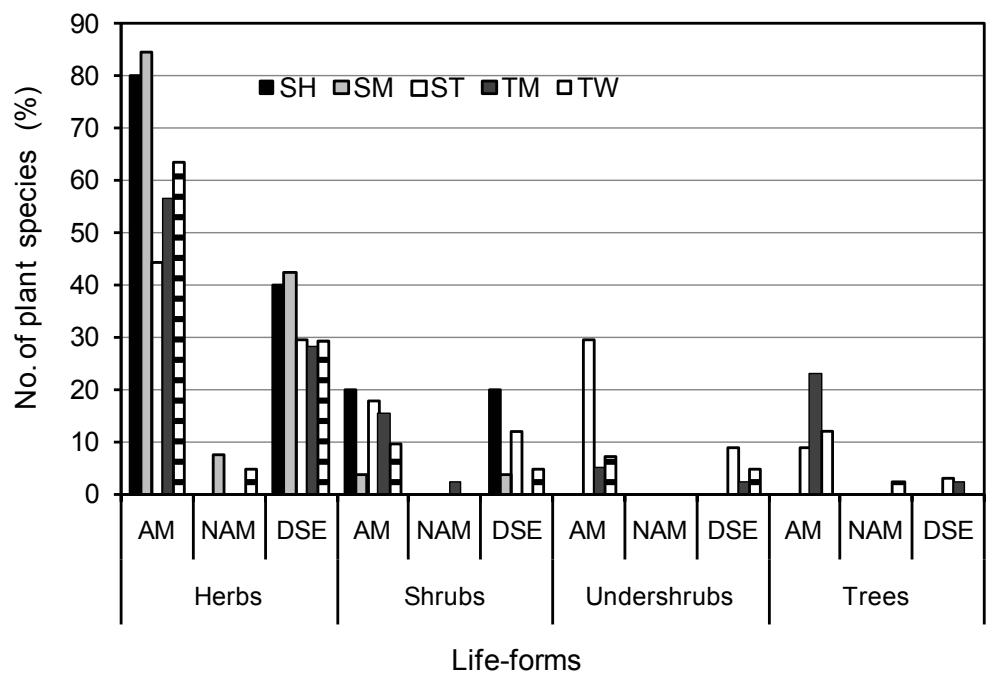

Fig. 3. The occurrence of arbuscular mycorrhizal (AM), non-mycorrhizal (NAM) and dark septate endophyte (DSE) fungal association in plants of various vegetation types and lifeforms in Velliangiri Hills. SH = Shola forest; SM = southern montane humid grassland; ST = southern tropical thorn forest; $\mathrm{TM}=$ tropical moist deciduous forest; $\mathrm{TW}=$ tropical wet evergreen forest 
AM fungal morphology in different vegetation types

Intermediate type AM morphology was frequent in plant species examined from different vegetation types (Table 2 and Fig. 4). The Paris-type AM morphology was maximum $(13 \%)$ in plants from the SM and minimum $(2.94 \%)$ in plants from the ST (Fig. 4). In general, intermediate type AM was more frequent (57\%) than the typical Arum- and Paris-types (34\% to 5\%) in different vegetation types (Table 2).

\section{Extent of AM and DSE fungal association}

Total root length colonisation (\%RLTC) ranged between $14.78 \%$ (Plantago erosa, Plantaginaceae) and $82.95 \%$ (Neolitsea scrobiculata, Lauraceae) and was significantly different between plant taxa $\left(X_{146}^{2}=203.617 ; \mathrm{P}<0.01\right)$. The percentage root length with hyphae $(\% \mathrm{RLH})$ ranged between $<1 \%$ (Emilia scabra, Asteraceae) and 46.91\% (Panicum sparsicomum, Poaceae), hyphal coils (\%RLHC) ranged between $1.94 \%$ (Solanum grandiflorum, Solanaceae) and $70.49 \%$ (Exacum sp., Gentianaceae), arbuscules / arbusculate coils (\%RLA/AC) ranged between $<1 \%$ (Persicaria barbata, Polygonaceae) and $54.29 \%$ (Bridelia stipularis, Euphorbiaceae), vesicles (\%RLV) ranged between $<1$ (Emilia sp., Asteraceae) and 21.52\% (Helichrysum sp., Asteraceae) (Table 3 and Fig. 1). Significant differences $(\mathrm{P}<0.01)$ existed between species for AM fungal variables such as \%RLH $\left(X^{2}{ }_{146}=193.471\right), \% R L V\left(X^{2}{ }_{146}=197.152\right), \% R L A\left(X^{2}{ }_{146}=\right.$ 192.847), and \%RLHC $\left(X^{2}{ }_{146}=202.510\right)$. In contrast, the differences for plant species among different forest types for \%RLH $\left(X^{2}{ }_{4}=1.785\right)$, \%RLV $\left(X^{2}{ }_{4}=\right.$

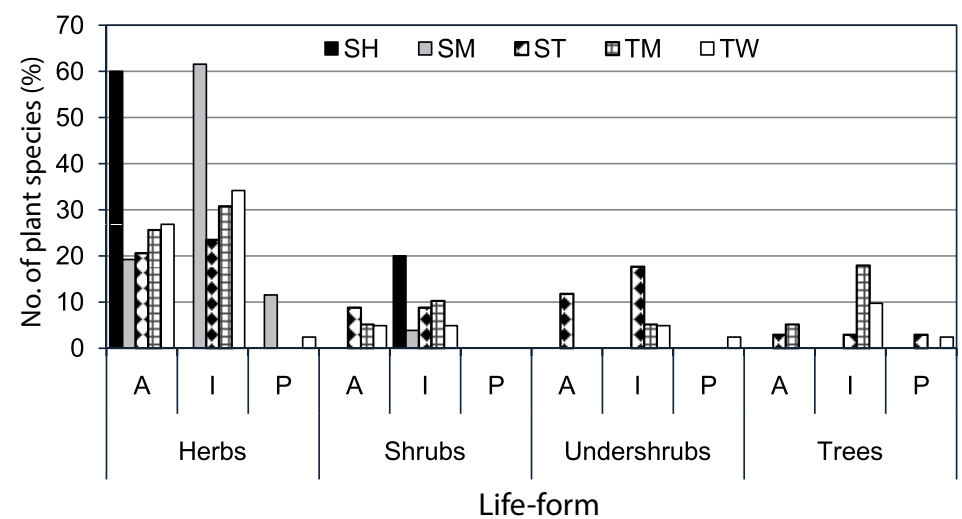

Fig. 4. Incidence of arbuscular mycorrhizal morphological types in plant species of various life-forms and vegetation types of Velliangiri Hills. SH = Shola forest; SM = southern montane humid grassland; ST = southern tropical thorn forest; TM = tropical moist deciduous forest; TW = tropical wet evergreen forest. $\mathrm{A}=$ Arum-; $\mathrm{P}=$ Paris-; $\mathrm{I}=$ intermediate type 
$0.611), \%$ RLHC $\left(X_{4}^{2}=3.107\right), \% \operatorname{RLTC}\left(X_{4}^{2}=1.953\right)$ are not significant $(\mathrm{P}>0.05)$. Nevertheless, significant differences existed for \%RLA/AC $\left(X_{4}^{2}=10.917 ; \mathrm{P}<0.05\right)$ among vegetation types. Average total colonisation was higher (50.34\%) in plants from tropical moist deciduous forests compared to other vegetation types.

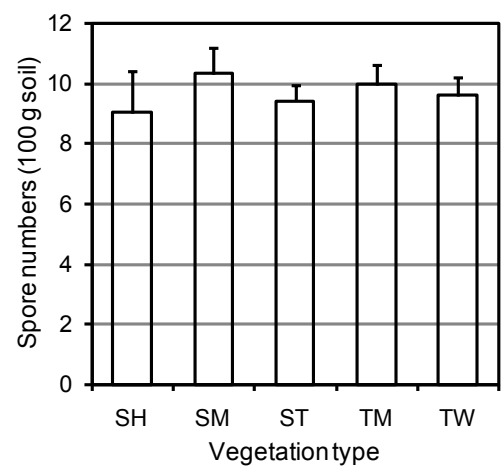

Fig. 5. Average arbuscular mycorrhizal spore numbers in different vegetation types of Velliangiri Hills. $\mathrm{SH}=$ Shola forest; $\mathrm{SM}=$ southern montane humid grassland; ST = southern tropical thorn forest; $\mathrm{TM}=$ tropical moist deciduous forest; $\mathrm{TW}=$ tropical wet evergreen forest. The error bars indicate $\pm 1 \mathrm{SE}$

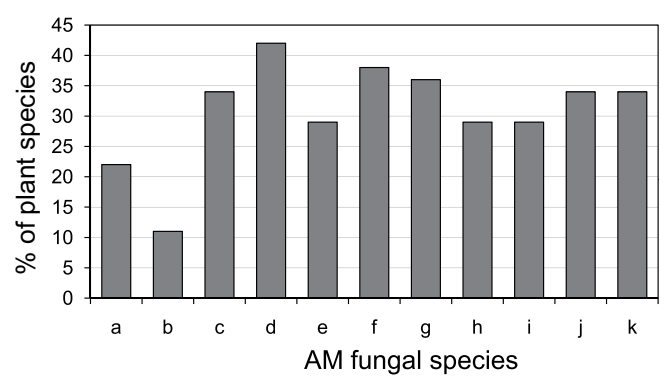

Fig. 6. The spore frequency of arbuscular mycorrhizal (AM) fungal species in rhizosphere of plants in different vegetation types of Velliangiri Hills. AM species code: $\mathrm{a}=$ Acaulospora scrobiculata, $\mathrm{b}=$ Gigaspora sp., $\mathrm{c}=$ Glomus aggregatum, $\mathrm{d}=$ Funneliformis geosporum, $\mathrm{e}=$ Glomus magnicaulis, $\mathrm{f}=$ Sclerocystis rubiformis, $\mathrm{g}=$ Sclerocystis sinuosa, $\mathrm{h}=$ Sclerocystis taiwaniensis, $\mathrm{i}=$ Glomus viscosum, $\mathrm{j}=$ Scutellospora heterogama, $\mathrm{k}=$ Scutellospora calospora

The extent of DSE fungal colonisation (\%RLDTC) ranged between $<1 \%$ (Ageratum houstonianum, Helichrysum sp., Asteraceae; Catunaregam spinosa, Rubiaceae; Mukia leiosperma, Cucurbitaceae; Oxalis corniculata, Oxalidaceae; Scutellaria sp., Labiatae) and 46.11\% (Mangifera indica, Anacardiaceae) (Table 3). Eighty-eight percent of the plant species had DSE fungal colonisation levels between 10 and $20 \%$. The percentage root length with DSE fungal hyphae (\%RLDH) ranged from $<1 \%$ (Strobilanthes sp., Acanthaceae; Ageratum houstonianum, Asteraceae; Catunaregam spinosa, Rubiaceae; Mukia leiosperma, Cucurbitaceae; Strobilanthes sp., Acanthaceae; Vigna unguicula$\mathrm{ta}$, Papilionaceae) to $28.10 \%$ ( $\mathrm{Cy}$ noglossum zeylanicum, Boraginaceae). Similarly, the root length with microsclerotia (\%RLMi) ranged from $<1 \%$ (Catunaregam spinosa, Rubiaceae; Fimbristylis strigosa, Fimbristylis ovata, Cyperaceae; Helichrysum sp., Asteraceae; Leucas sp., Scutellaria sp., Labiatae; Oxalis corniculata, Oxalidaceae, Vigna unguiculata, Papilionaceae) to $44 \%$ (Mangifera indica, Anacardiaceae) (Table 3 and Fig. 2). DSE fungal variables like \%RLDH $\left(X_{4}^{2}=\right.$ 5.482), \%RLDMi $\left(X_{4}^{2}=2.047\right)$ and $\operatorname{RLDTC}\left(X_{4}^{2}=0.917\right)$ did not differ 
significantly $(\mathrm{P}>0.05)$ among forest types, but differed significantly $(\mathrm{P}<0.01)$ among species (RLDH, $X^{2}{ }_{146}=198.539 ; \% R L D M i, X^{2}{ }_{146}=194.047$ and \%RLDTC, $\left.X^{2}{ }_{146}=203.541\right)$. Though, Pearson's correlation analysis indicated the lack of correlation between \%RLDTC and \%RLTC $(r=0.149 ; \mathrm{P}>0.05 ; \mathrm{n}=147)$, the association was positive as the probability of co-occurrence frequency (p_gt) was greater than the observed frequency for these fungal types $\left(\mathrm{p} \_g t=0.00076\right)$.

\section{AM fungal spore numbers in different vegetation types}

The AM fungal spore numbers in the plant root zones from different vegetations ranged from 2 (Gynostemma pentaphyllum, Cucurbitaceae) to 19 spores / $100 \mathrm{~g}$ soil (Conyza leucantha, Asteraceae) (Table 3). There was no significant difference among various vegetation types on spore numbers $\left(X^{2}{ }_{4}=\right.$ $0.183)$, however, spore numbers differed significantly among species $\left(X^{2}{ }_{146}=\right.$ 202.675). Spore numbers were not related to \%RLTC $(\mathrm{r}=-0.039 ; \mathrm{P}>0.05 ; \mathrm{n}=$ 147 ) and average AM fungal spore numbers were higher (10.31 spores / 100 $\mathrm{g}$ soil) in the SM (Fig. 5). Eleven AM fungal spore morphotypes were isolated and identified which included one species in Acaulospora (Acaulospora scrobiculata Trappe), Funneliformis [Funneliformis geosporum (T. H. Nicolson et Gerd.) C. Walker et A. Schüßler], Gigaspora sp., two in Scutellospora [Scutellospora calospora (T. H. Nicolson et Gerd.) C. Walker et F. E. Sanders and Scutellospora heterogama (T. H. Nicolson et Gerd.) C. Walker et F. E. Sanders], three in Glomus (Glomus aggregatum N. C. Schenck et G. S. Sm, Glomus viscosum T. H. Nicolson, Glomus magnicaule I. R. Hall) and Sclerocystis (Sclerocystis taiwaniensis C. G. Wu et Z. C. Chen, Sclerocystis rubiformis Gerd. et Trappe, Sclerocystis sinuosa Gerd. et B. K. Bakshi). Among the different AM spore morphotypes, F. geosporum was found associated with maximum number of plant species and Gigaspora sp., was found associated with minimum number of plant species (Fig. 6). Spores of Scutellospora heterogama were the most frequent $(42 \%)$ in the SM and TM (Fig. 7). In the ST and SH, F. geosporum (50\% and 80\%) were more frequent. Sclerocystis rubiformis and $S$. sinuosa were the most frequent species occurring in TM and TW, respectively. Across vegetation types, F. geosporum was the most frequent species occurring in the rhizospheres of $47 \%$ of the plant species, whereas spores of Gigaspora sp. were infrequent occurring in the rhizospheres of around 13\% of the plant species (Fig. 7). Of the 55 AM fungal species pairs that occurred with different plant species $24 \%$ were positive and $38 \%$ were negative and $38 \%$ were random associations (Fig. 8 ). The species contributing to this high randomness were Gigaspora sp., G. aggregatum, and A. scrobiculata. Around $50 \%$ of the associations of G. magnicaulis and G. viscosum with other AM fungal species were negative (Fig. 8). 


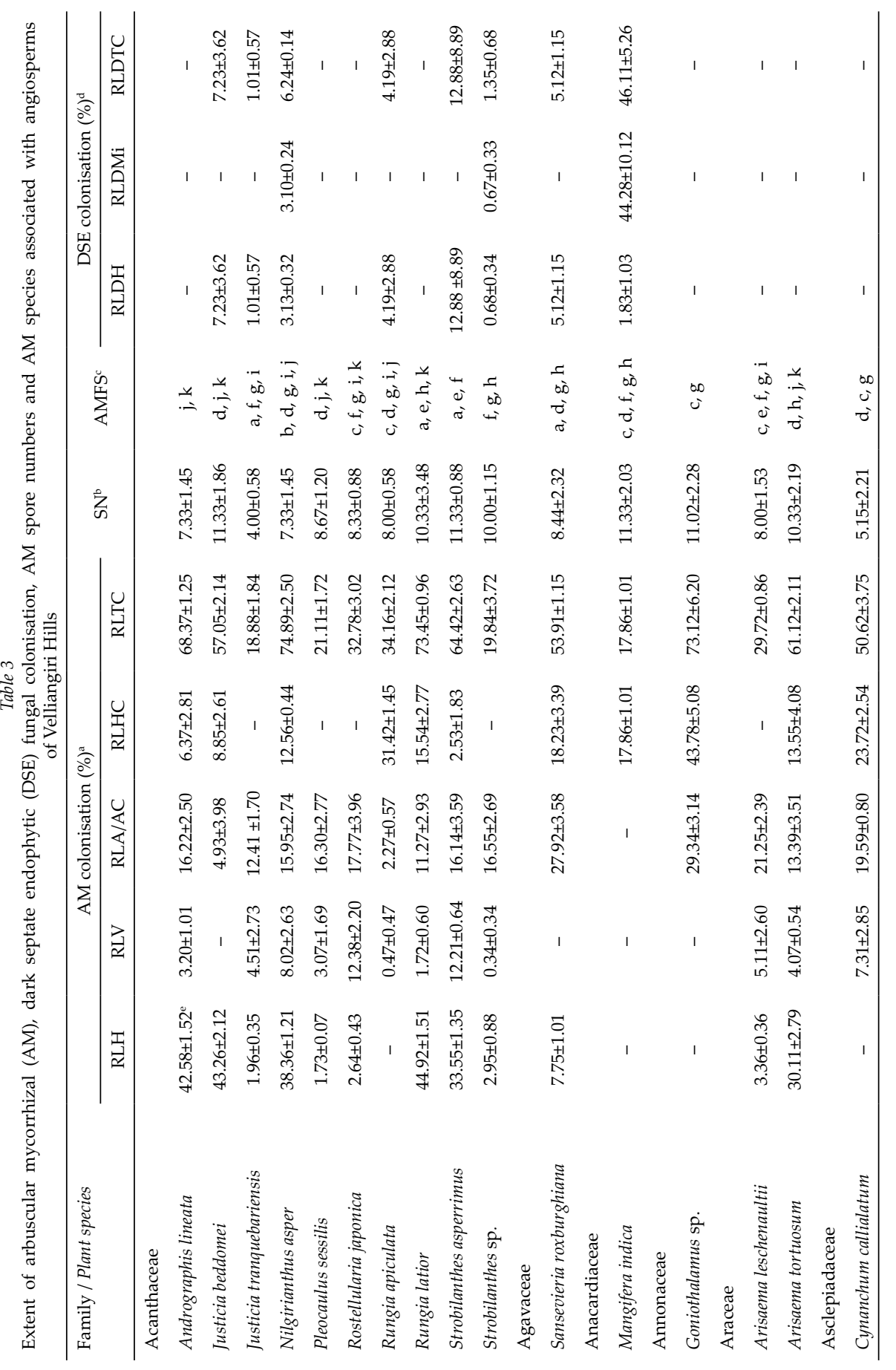




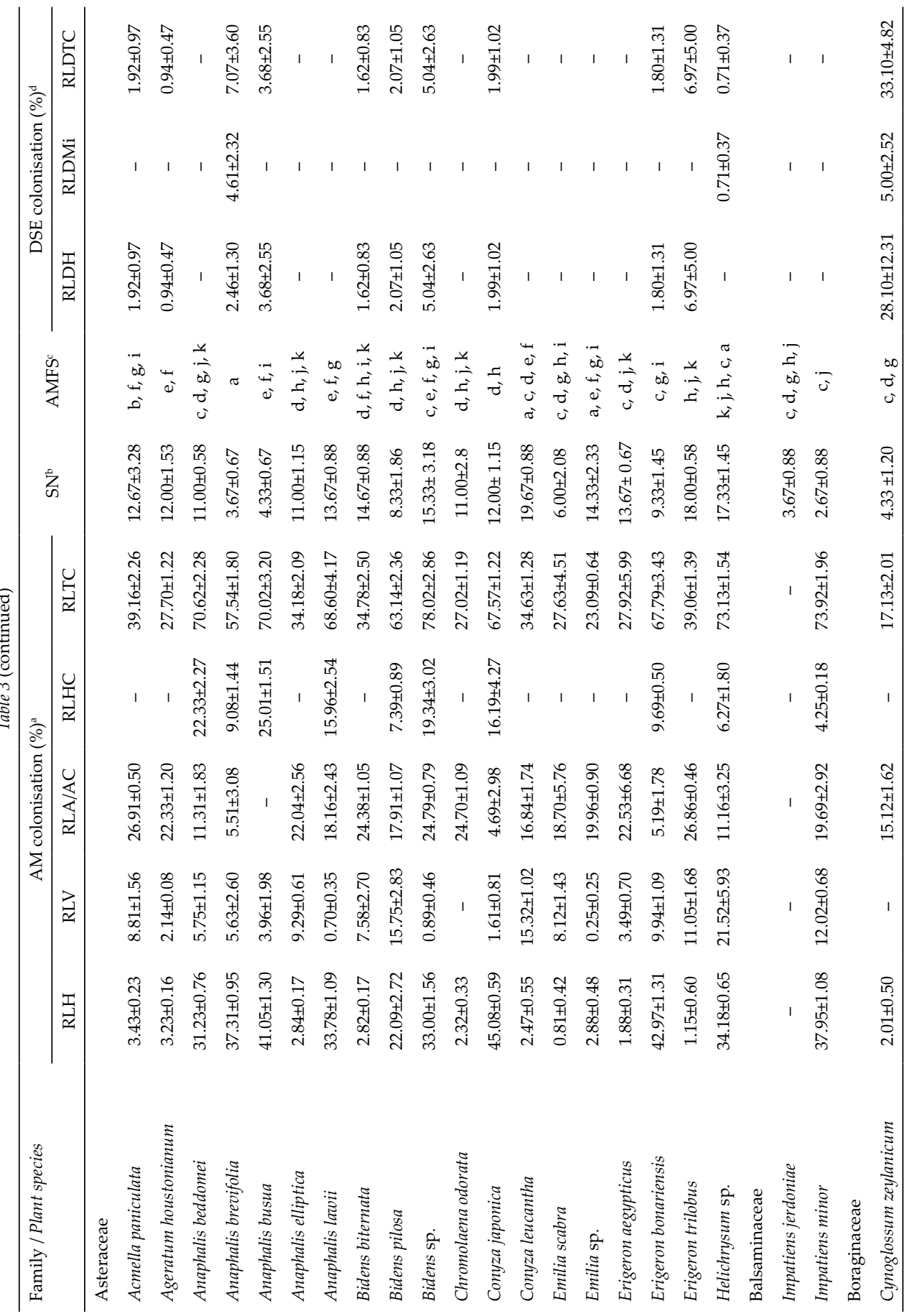




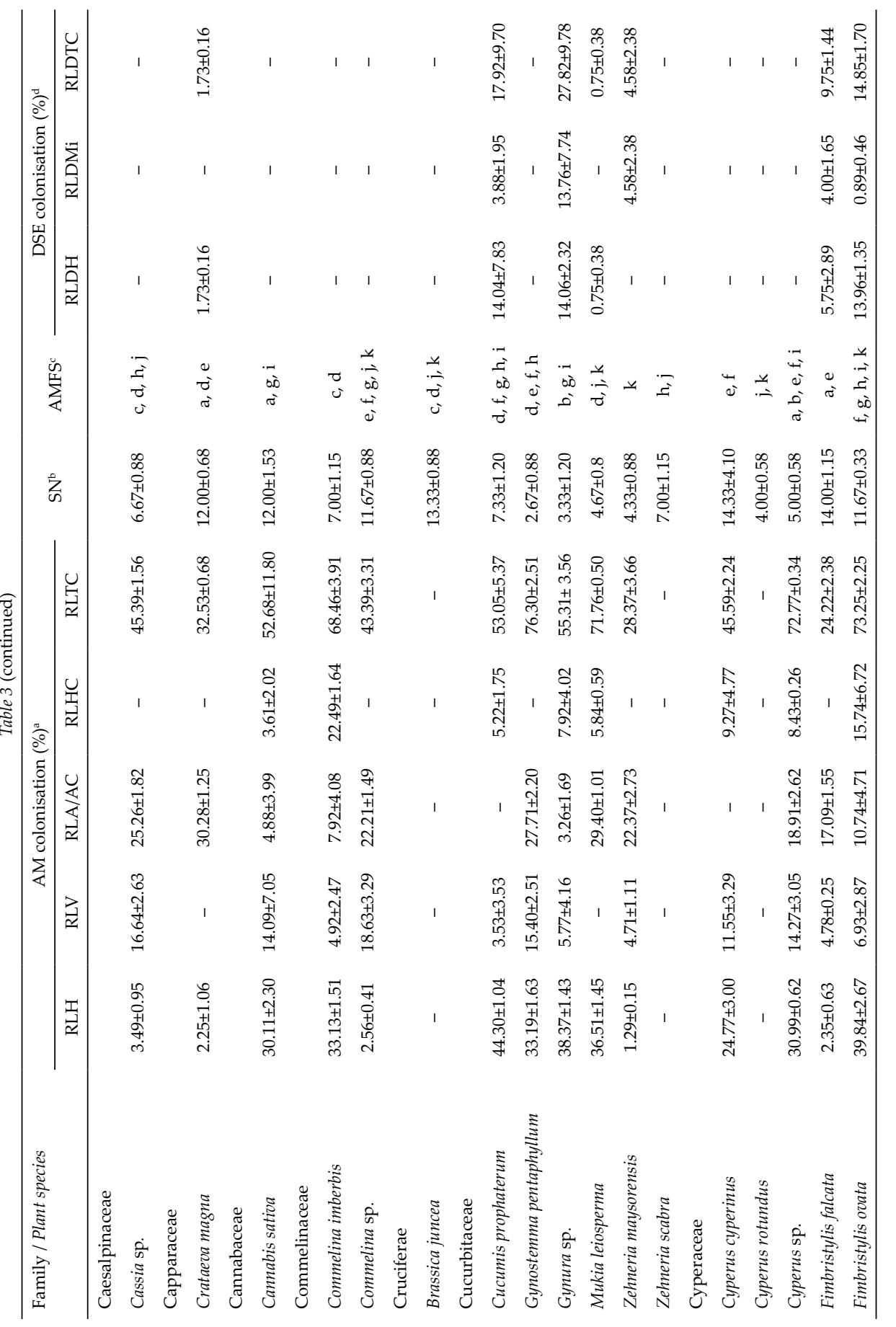




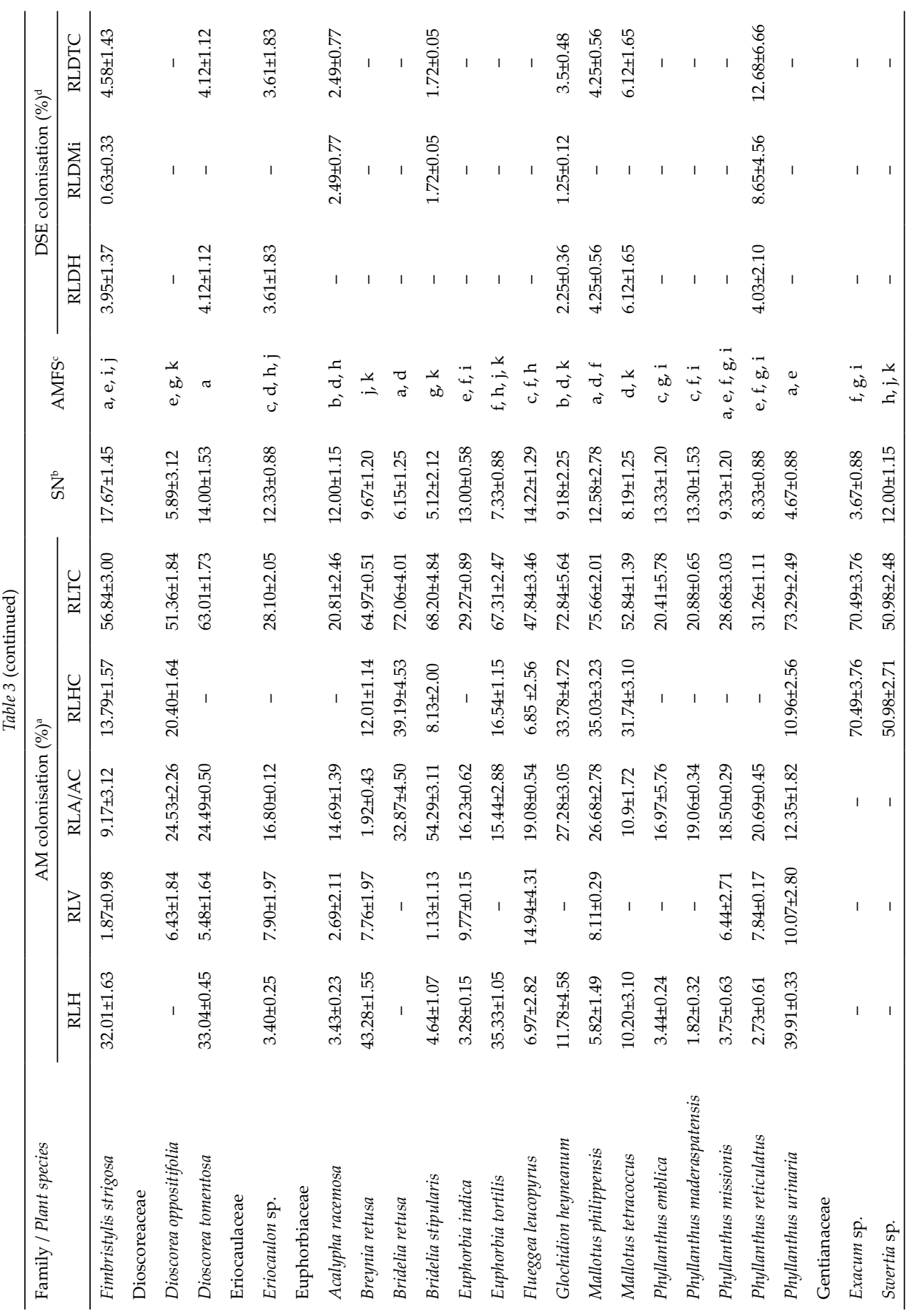




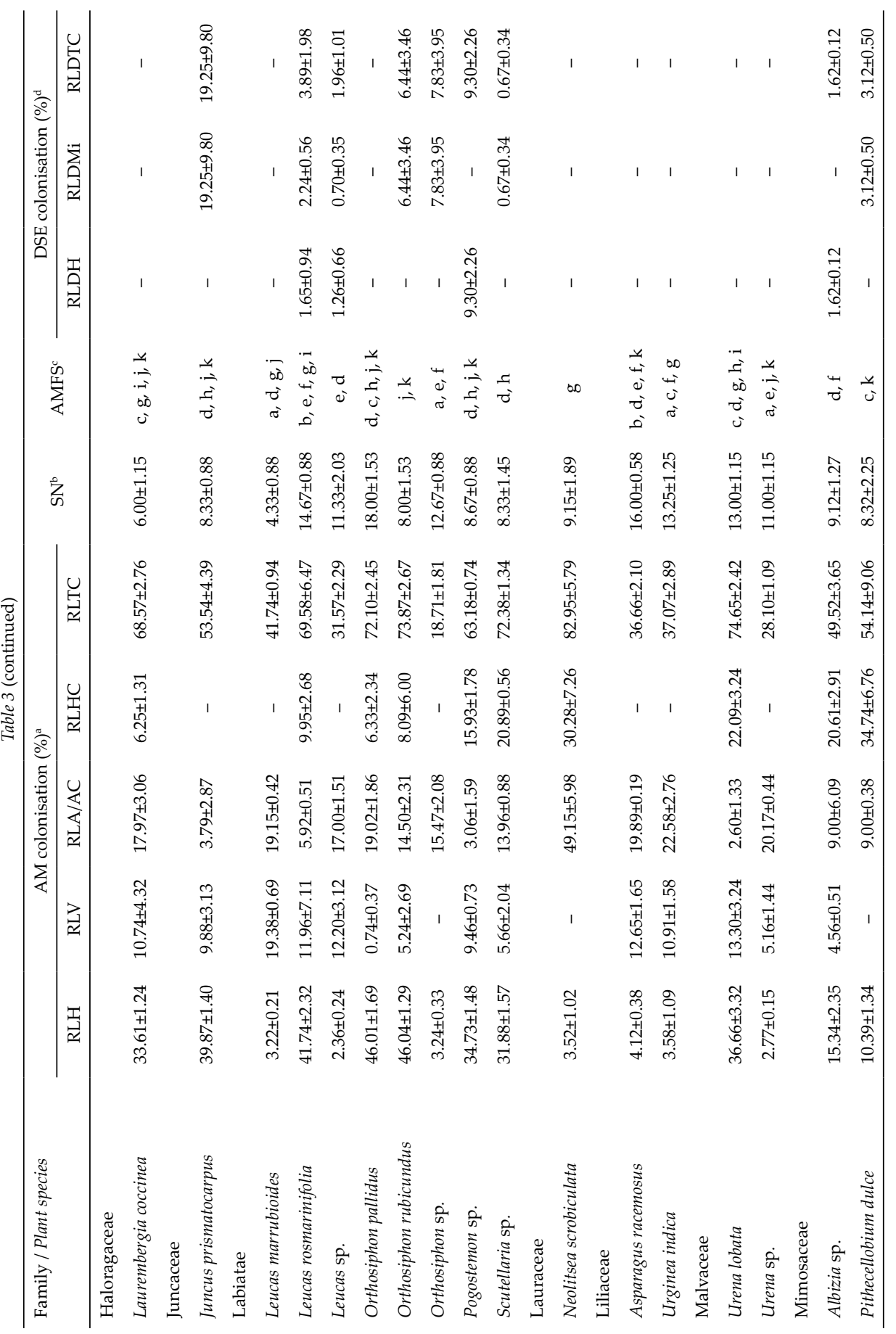




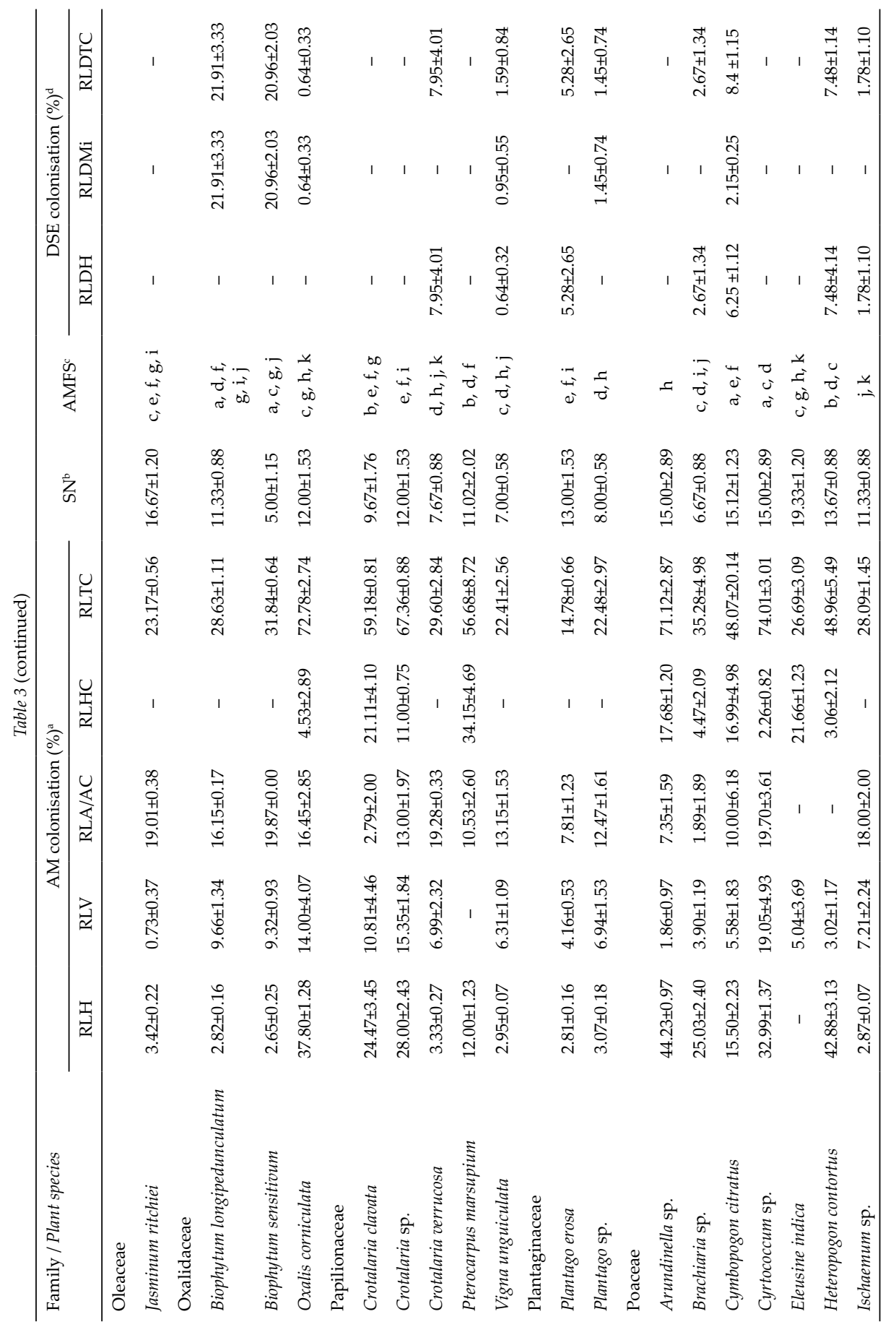




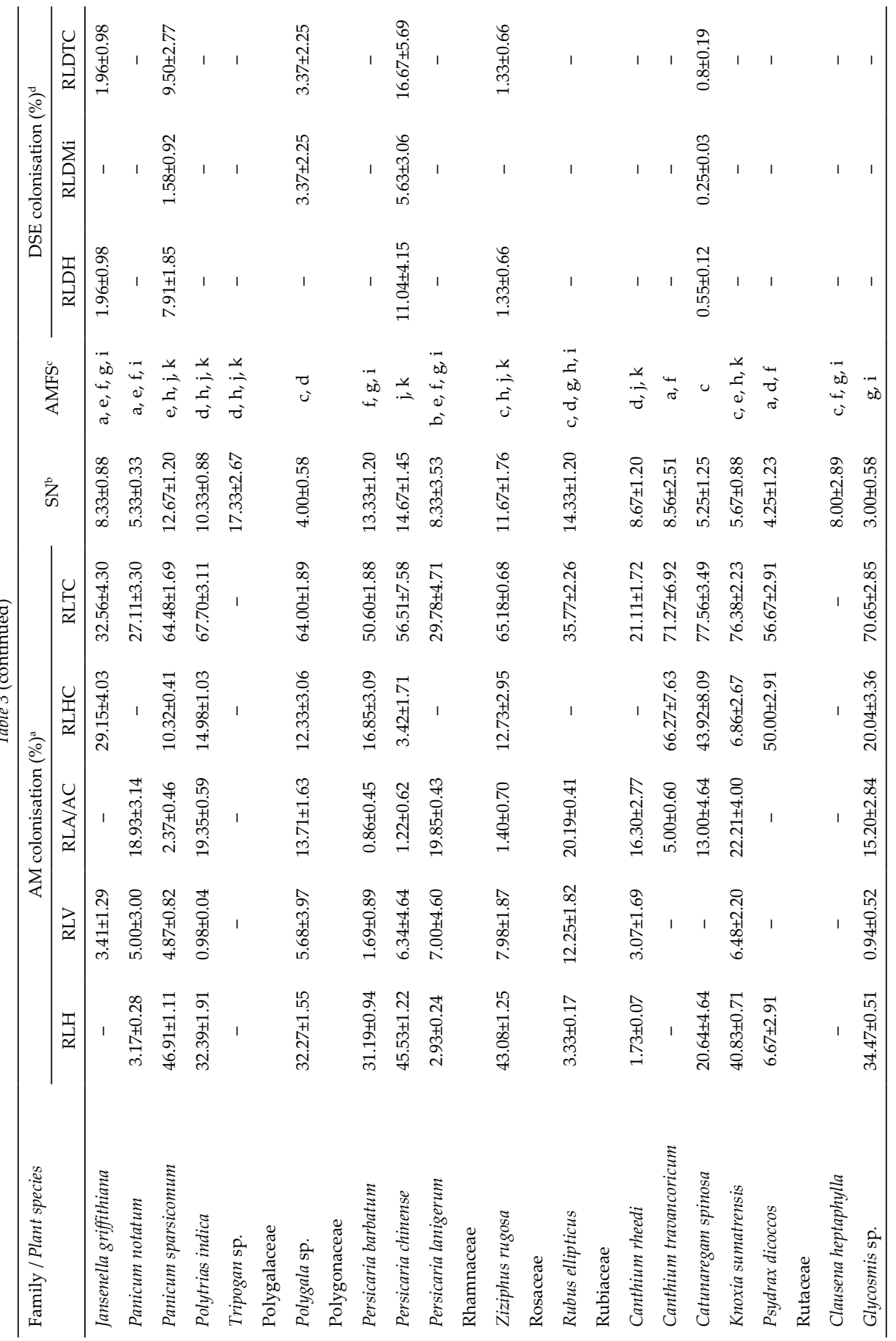




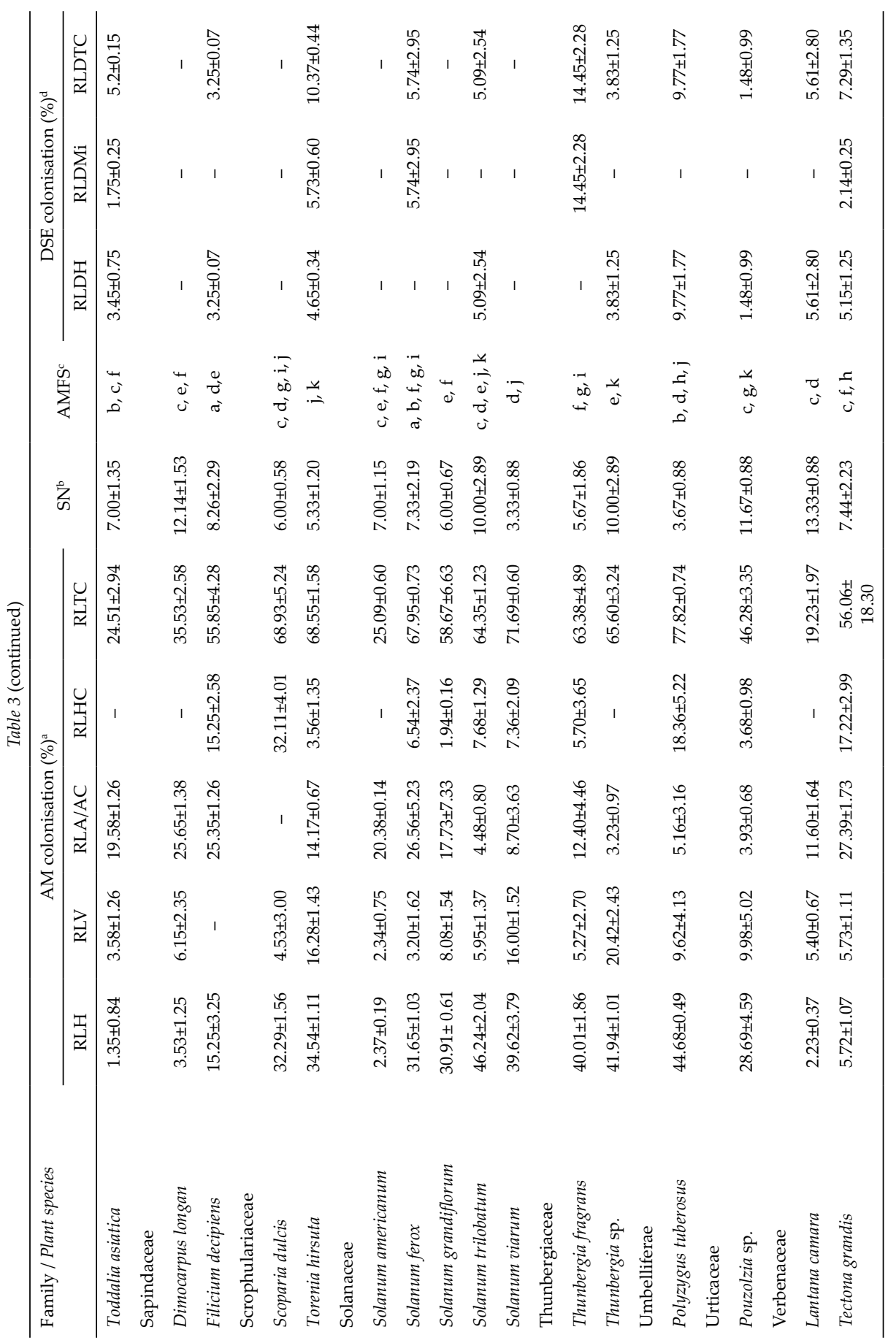




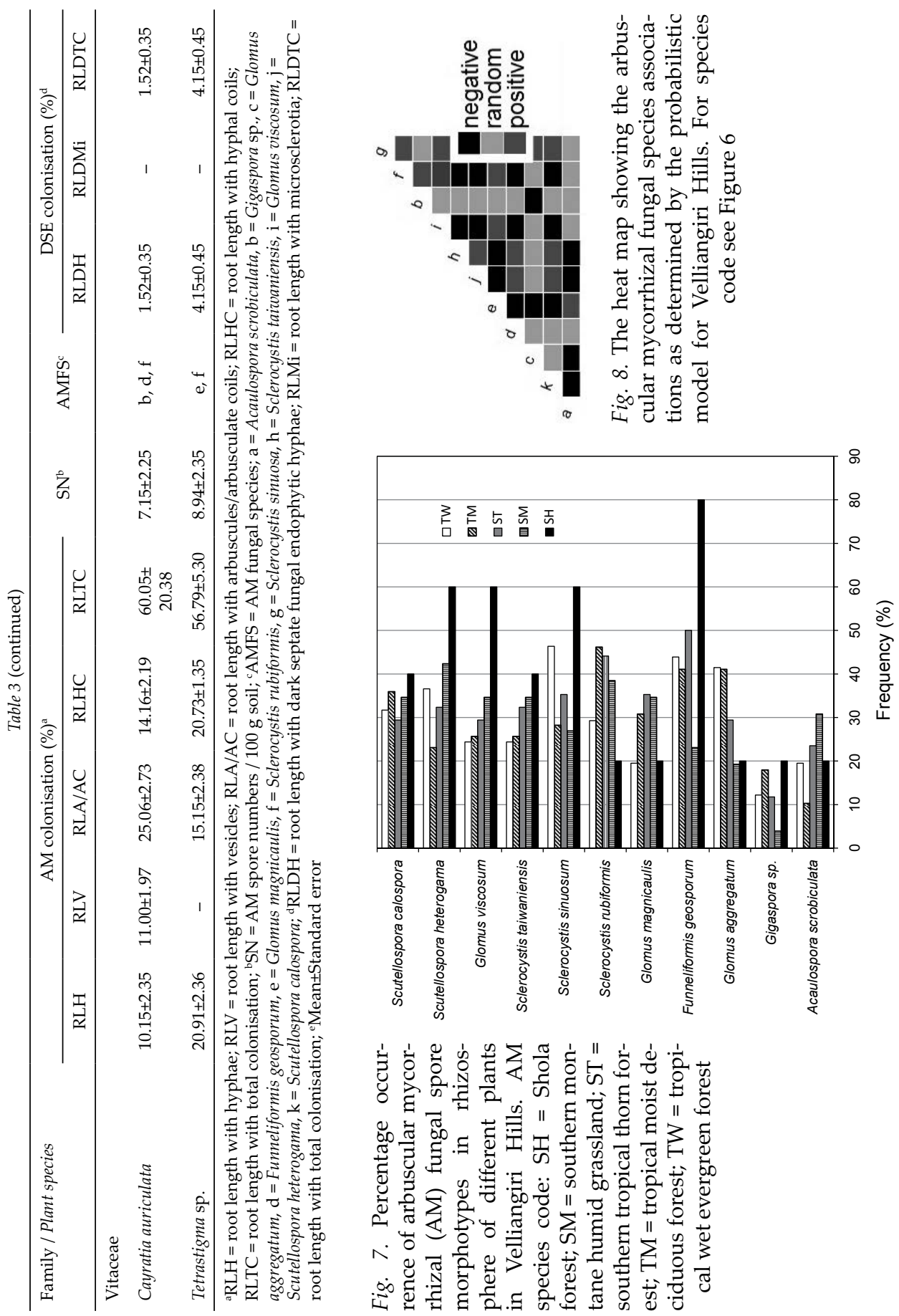




\section{DISCUSSION}

Arbuscular mycorrhizal associations were found in most of the plant taxa examined from different vegetation types of Velliangiri Hills. This concurs with the observations of Wang and Qiu (2006) and Brundrett (2009) who indicated the widespread occurrence of mycorrhizae among vascular plants. Further, AM and DSE fungal associations have been reported to our knowledge in 61 and 42 plant species, respectively, for the first time. High competition for resources and low nutrient status of the soils could have resulted in the high prevalence of AM in plants of different vegetation types. Significant differences both in \%RLTC and root length with different AM fungal structures indicate the variation in the dependence of plant species on AM fungi, as a positive correlation between AM fungal colonisation levels and plant-mycorrhizal dependency has been reported in P-deficient soils (Smith et al. 2011).

\section{Occurrence of AM and DSE fungi in various vegetation types}

Ninety-two percent of plants examined from grasslands were colonised by AM fungi which is almost similar to the findings of Li et al. (2004) who also reported AM in $95 \%$ of plant species they examined in valley-type savanna in southwest China and $82 \%$ (18/22) of the grassland plant species examined by Gai et al. (2006) from southern Tibet were mycorrhizal. The presence of AM in $97 \%$ of the plant species from the tropical moist deciduous forest is consistent with the findings of Allen et al. (1998) who reported AM association in all the plant species they examined from a Mexican tropical deciduous forest.

The plants that lacked mycorrhizae in the present study belonged to Balsaminaceae, Cruciferae, Cucurbitaceae, Cyperaceae and Poaceae. Of these, except Cruciferae, all other families have been reported as mycorrhizal (Brundrett 2009, Muthukumar et al. 2004, Wang and Qiu 2006). Brassica juncea examined from tropical wet evergreen forest lacked mycorrhizal associations in the present study. Nevertheless, AM characterised by the presence of Glomustype vesicles and hyphae but not arbuscules have been reported in other species of Brassica like Brassica napus and Brassica oleracea (Harley and Harley 1987, Tester et al. 1987, Vierheilig et al. 2000). A functional association with abundant arbuscules in Brassica has never been found so far. The other nonmycorrhizal family Cyperaceae is no longer considered so, but the mycorrhizal status of its members can be greatly influenced by environmental factors (Brundrett 2009, Muthukumar et al. 2004). Cyperus rotundus has been reported to be both mycorrhizal (Muthukumar and Udaiyan 2002, Muthukumar et al. 1997) and non-mycorrhizal (Koske and Gemma 1992) in different studies. 
The presence of DSE fungal association in $62 \%$ of the plant species in the southern tropical thorn forest corroborates the observations of Fracchia et al. (2009) who reported DSE association in $75 \%$ of the plant species they examined from Chaco Serrano woodland colonised by DSE fungi. All the plant species harbouring DSE fungi were also mycorrhizal, which is in line with the observations of Fracchia et al. (2009), Sathiyadash et al. (2010) and Uma et al. (2010), where dual colonisation of AM and DSE fungi has been reported. The present studies, as well as the other studies cited above, clearly suggest that DSE fungal associations in various vegetation types exist as a backup system and tend to take over the functions of AM fungi under conditions unfavourable for AM fungi (Jumpponen and Trappe 1998).

\section{$A M$ and DSE fungal association in plant life-forms}

The widespread occurrence of AM in herbs of thorn and deciduous forests agrees with findings of Yamato and Iwasaki (2002), where all the herbaceous understory plants examined from Japanese deciduous broad-leaved forests were mycorrhizal. Our findings of high incidence of DSE fungal association in herbs compared to other life-forms examined from different vegetations is in accordance with other studies where high prevalence of DSE association has been reported in herbs (Muthukumar et al. 2006, Schmidt et al. 2008). Trees examined from the different vegetation types in the present study were invariably colonised by AM fungi similar to the observations of Fracchia et al. (2009) and Uma et al. (2012), where all the tree species examined from Chaco Serrano woodlands from central Argentina and South India, respectively, were mycorrhizal. A high incidence of mycorrhizae in tree species has also been reported by Onguene and Kuyper (2001) from south Cameroon (74\%) and McGuire et al. (2008) from central Guyana (94\%). Generally, trees have a very sparse root system so to increase the surface for absorption of nutrients trees tend to depend on AM fungi (Phillips and Fahey 2006). This may be the reason for high incidence of AM association of trees in the present study. Shrubs from all the vegetation types examined, except those from tropical moist deciduous forests were mycorrhizal. This is in line with the observations of Fracchia et al. (2009), who also reported the presence of AM in all the shrubs they examined from Chaco Serrano woodlands, Argentina. However, Fontenla et al. (2001) found only 86\% of shrubs in Patagonian steppe, Argentina to be mycorrhizal. In general, the frequent occurrence of AM in plant roots from all the vegetation types shows the inability of non-mycorrhizal plants to compete with mycorrhizal plants in these well-established ecosystems (Smith and Read 2008). 


\section{Extent of AM and DSE fungal association}

Generally, it is hypothesised that plants with a more efficient root system are less dependent on mycorrhizas the plants with less efficient root system. Nevertheless, this hypothesis was recently rejected by Maherali (2014) and the results of the present study also supports this as the calculated frequency of occurrence of AM tended to be almost similar in dicots (96.46\%) with sparse root system and monocots (93.93\%) with highly efficient fibrous root system. Likewise, mean colonisation levels of dicots $(52 \%)$ were only slightly higher than those of monocots (48\%). This suggests that in highly competitive ecosystems like those studied here even the moderately or low mycorrhizal plant species tend to depend on AM fungi. The extent of DSE fungal colonisation in most of plant species was $<20 \%$, which is in accordance with Rains et al. (2003) who also reported low levels of DSE fungal colonisation (25\%) in the epiphytic and terrestrial plants they examined from the Costa Rican cloud forest. The lack of correlation between the root length colonised by AM and DSE fungi suggest that these two fungal groups do not significantly influence each other within roots. Moreover, the positive association of these fungal groups as evidenced by the probabilistic model indicates that these fungal types could coexist in a wide range of plant species and in different ecosystems.

\section{AM fungal morphology in vegetation types}

In this study AM morphology dominated by intermediate type is reported for the first time in 64 plant species. The dominance of the intermediate type AM morphology in the present study is consistent with the findings of Uma et al. (2010), who observed that $80 \%$ (32/40) of the gingers and spiral gingers are intermediate type AM. The presence of the Arum-type morphology in only 33\% of the tropical wet evergreen forest plant species is consistent with the findings of Matekwor Ahulu et al. (2005), where 33\% of evergreen species they examined had Arum-type AM. But the occurrence of Paris-type $\mathrm{AM}$ in $67 \%$ of the evergreen species of a mixed pine forest of Central Honsu, Japan (Matekwor Ahulu et al. 2005) does not agree with the present study where only $8 \%$ of the plants from tropical wet evergreen forests formed typical Paris-type AM. Instead, the majority of plants from this vegetation type formed intermediate type AM. This suggests that under low light intensity in the evergreen forests, plants tend to grow slowly and favour formation of Paris-type colonisation (Brundrett et al. 1990). When compared to other forest types (50\%) species of the Southern montane humid grassland had Paristype AM. This is in accordance with the findings of Muthukumar et al. (2006), who reported Paris-type AM in $71 \%$ of the plant species they examined from 
grasslands of Western Ghats. At high altitudes, plant growth tends to slow down due to various environmental factors, which may favour the formation of Paris-type AM in plants at high altitudes (Matekwor Ahulu et al. 2005).

\section{AM fungal spore characteristics of the study sites}

Spore numbers in present study were low (2 to 19 spores per $100 \mathrm{~g}$ soil) compared to 135 to 150 spores per $100 \mathrm{~g}$ soil reported by Suchitra et al. (2012) for different soil types in Tamilnadu, India and 66 to 1,308 spores per $100 \mathrm{~g}$ soil reported by Lakshmipathy et al. (2011) from different land use systems in the Nilgiris biosphere reserve of the Western Ghats, southern India. Zangaro et al. (2013) also reported a high AM fungal spore numbers ranging from 20 to 150 spores per $150 \mathrm{~g}$ soils from soils of an Atlantic forest biome in southern Brazil. The low density of AM fungal spores documented the present study, however, is consistent with the reports from humid tropical forests where spore numbers tend to be low or infrequent (Fischer et al. 1994, Janos 1980). The observation of the present and other studies clearly indicates that in vegetation types where root growth occurs throughout the year AM colonisation of new roots can occur through the extraradical hyphae in the soil or the hyphae emerging from mycorrhizal roots. Hence, in such ecosystems, the spore populations may be small despite high root colonisation levels (Muthukumar et al. 2003, Zandavalli et al. 2008). In this study, spores of AM fungi were present in the rhizospheres of NM plants. In natural vegetations, AM and non-mycorrhizal plants grow in close proximity and the roots of these two often overlap. Therefore, the AM spores in the rhizosphere a plant could be contributed by the coexisting companion AM plants (Muthukumar and Udaiyan 2000a, b).

A total of 11 AM fungal morphospecies were identified, which is similar to the observations of Sathiyadash et al. (2010) who also observed spores of 11 AM fungal species in association with South Indian Grasses. However, Muthukumar and Udaiyan (2000a) reported 6-22 morphospecies per site from the Western Ghats region of South India. Arbuscular mycorrhizal fungal spores belonging to Glomus dominated species diversity, which is in accordance with the observations that species of Glomus dominate tropical soils (Devi et al. 2017, D'Souza and Rodrigues 2013, Kumar and Adholeya 2016, Sathiyadash et al. 2010, Suchitra et al. 2012). The lack of correlation between spore numbers and \%RLTC suggests that root colonisation could be initiated by propagules other than spores like AM fungal hyphae in the soil or mycorrhizal roots of the same plant or conspecifics (Smith and Read 2008). An interesting observation made in the present study is the different types of associations among AM fungi. Although, AM fungal community composition and the role of vari- 
ous factors influencing it are well known, the interaction between different AM fungal species has been largely ignored (Alguacil et al. 2016, Gosling et al. 2013). As obligate biotrophs the distribution of AM fungi depends on the host plants (Álvarez-Sánchez et al. 2012). Moreover, AM fungi have been shown to possess low host specificity as experimental evidences do indicate that an AM fungus can colonise a wide range of plant species (Álvarez-Sánchez et al. 2012). Nevertheless, in the present study most of the AM fungal pairs (72\%) had either positive or negative associations. This suggests the existence of certain level of host preference as certain fungi tend to associate with a large number of plant species and sporulate abundantly, while others tend to more restricted in their association and sporulation (Álvarez-Sánchez et al. 2012, Vandenkoornhuyse et al. 2002).

\section{CONCLUSIONS}

Arbuscular mycorrhizal and DSE fungal association were found to be widespread in plant species from different vegetation types in Velliangiri Hills. Moreover, the report of AM and DSE fungal associations in several plants for the first time clearly indicates that many plants in natural vegetations are yet to be examined for their fungal associations. The widespread occurrence of AM and DSE in rare and economically important plant species, emphasises the potential for their exploitation in plant production systems. The number of AM fungal spores and their diversity was less compared to reports from other vegetations worldwide. This study for the first time shows that there could be different types of associations between co-occurring species in AM fungal communities. Further, experimental studies are required to determine the effects of the fungal inoculants in the conservation of these rare and economically important plant species.

Acknowledgements - We thank Prof. Christos Mammides, Guangxi Key Laboratory of Forest Ecology and Conservation, College of Forestry, Guangxi University, Daxuedonglu 100, Nanning, 530004, China for his help in probabilistic co-occurrence analysis. We also sincerely thank the anonymous reviewers whose suggestions and critical comments greatly helped in improving an earlier version of this paper.

\section{REFERENCES}

Alguacil, M. D. M., Torres, M. P., Montesinos-Navarro, A. and Roldán, A. (2016): Soil characteristics driving arbuscular mycorrhizal fungal communities in semiarid Mediterranean soils. - Appl. Environ. Microbiol. 82: 3348-3356. https://doi.org/10.1128/ aem.03982-15 
Allen, E. B., Rincon, E., Allen, M. F., Perez-Jimenez, A. and Huante, P. (1998): Disturbance and seasonal dynamics of mycorrhizae in a tropical deciduous forest in Mexico. Biotropica 30: 261-274. https://doi.org/10.1111/j.1744-7429.1998.tb00060.x

Álvarez-Sánchez, J., Johnson, N., Antoninka, A., Chaudhary, V., Lau, M., Owen, M., Sànchez-Gallen, I., Guadarrama, P. and Castillo, S. (2012): Large-scale diversity patterns in spore communities of arbuscular mycorrhizal fungi. - In: Pagano, M. C. (ed.): Mycorrhiza: occurrence and role in natural and restored environments. Nova, New York, pp. 29-47.

Balasubramaniam, V. and Murugesan, M. (2004): A note on the commercially exploited medicinal plants of the Velliangiri hills, Coimbatore district, Tamilnadu. - Anc. Sci. Life 23: 9-12.

Berruti, A., Lumini, E., Balestrini, R. and Bianciotto, V. (2015): Arbuscular mycorrhizal fungi as natural biofertilizers: Let's benefit from past successes. - Front. Microbiol. 6: 1559. https://doi.org/10.3389/fmicb.2015.01559

Bray, R. H. and Kurtz, L. T. (1945): Determination of total, organic, and available forms of phosphorus in soils. - Soil Sci. 59: 39-45. https://doi.org/10.1097/00010694-19450100000006

Brundrett, M. C. (2009): Mycorrhizal associations and other means of nutrition of vascular plants: understanding the global diversity of host plants by resolving conflicting information and developing reliable means of diagnosis. - Plant Soil 320:37-77. https:// doi.org/10.1007/s11104-008-9877-9

Brundrett, M. and Kendrick, B. (1990): The roots and mycorrhizas of herbaceous woodland plants II. Structural aspects of morphology. - New Phytol. 114: 469-479. https://doi. org/10.1111/j.1469-8137.1990.tb00415.x

Brundrett, M. C., Murase, G. and Kendrick, B. (1990): Comparative anatomy of roots and mycorrhizae of common Ontario trees. - Can. J. Bot. 68: 551-578. https://doi. org $/ 10.1139 / \mathrm{b} 90-076$

D'Souza, J. and Rodrigues, B. F. (2013): Seasonal diversity of arbuscular mycorrhizal fungi in mangroves of Goa, India. - Int. J. Biodiv. 2013: 1-7. https://doi.org/10.1155/2013/196527

da Silva, I. R., de Souza, F. A., da Silva, D. K. A., Oehl, F. and Maia, L. C. (2017): Patterns of arbuscular mycorrhizal fungal distribution on mainland and island sandy coastal plain ecosystems in Brazil. - Microb. Ecol. 74(3): 654-669. https://doi.org/10.1007/ s00248-017-0979-x

Devi, S., Bhattacharya, P., Verma, P. and Verma, R. K. (2017): Diversity status of arbuscular mycorrhizal (AM) fungi in association with important NTFP species in tropical forests of central India. - Indian Forester 143: 364-369.

Dickson, S. (2004): The Arum-Paris continuum of mycorrhizal symbioses. - New Phytol. 163: 187-200. https://doi.org/10.1111/j.1469-8137.2004.01095.x

Dickson, S., Smith, F. A. and Smith, S. E. (2007): Structural differences in arbuscular mycorrhizal symbioses: more than 100 years after Gallaud, where next? - Mycorrhiza 17: 375-393. https://doi.org/10.1007/s00572-007-0130-9

Fischer, C. R., Janos, D. P., Perry, D. A., Linderman, R. G. and Sollins, P. (1994): Mycorrhiza inoculum potentials in tropical secondary succession. - Biotropica 26: 369-377. https:// doi.org/10.2307/2389230

Fontenla, S., Puntieri, J. and Ocampo, J. A. (2001): Mycorrhizal associations in the Patagonian steppe, Argentina. - Plant Soil 233: 13-29. 
Fracchia, S., Aranda, A., Gopar, A., Silvani, V., Fernandez, L. and Godeas, A. (2009): Mycorrhizal status of plant species in the Chaco Serrano Woodland from central Argentina. - Mycorrhiza 19: 205-214. https://doi.org/10.1007/s00572-009-0231-8

Gai, J. P., Cai, X. P., Feng, G., Christae, P. and Li, X. L. (2006): Arbuscular mycorrhizal fungi associated with sedges on the Tibetan plateau. - Mycorrhiza 16: 151-157. https://doi. org/10.1007/s00572-005-0031-8

Gosling, P., Mead, A., Proctor, M., Hammond, J. P. and Bending, G. D. (2013): Contrasting arbuscular mycorrhizal communities colonizing different host plants show a similar response to a soil phosphorus concentration gradient. - New Phytol. 198: 546-556. https://doi.org/10.1111/nph.12169

Griffith, D. M., Veech, J. A. and Marsh, C. J. (2016): Cooccur: probabilistic species co-occurrence analysis in R. - J. Stat. Softw. 69: 1-17. https://doi.org/10.18637/jss.v069.c02

Grilli, G., Urcelay, C., Galetto, L., John, D., Vasar, M., Saks, Ü., Jairus, T. and Öpik, M. (2015): The composition of arbuscular mycorrhizal fungal communities in the roots of a ruderal forb is not related to the forest fragmentation process. - Environ. Microbiol. 17: 2709-2720. https://doi.org/10.1111/1462-2920.12623

Harley, J. L. and Harley, E. L. (1987): A check-list of mycorrhiza in the British flora. - New Phytol. 105: 1-102. https://doi.org/10.1111/j.1469-8137.1987.tb00674.x

Henry, A. N., Chitra, V. and Balakrishnan, N. P. (1989): Flora of Tamil Nadu, India. Vol. 3. Botanical Survey of India, Coimbatore, India, $171 \mathrm{pp}$.

Henry, A. N., Kumar, G. R. and Chitra, V. (1987): Flora of Tamil Nadu, India. Vol. 2. - Botanical Survey of India, Coimbatore, India, $258 \mathrm{pp}$.

Jackson, M. L. (1971): Soil chemical analysis. - Prentice-Hall, New Delhi, India, 498 pp.

Janos, D. P. (1980): Vesicular-arbuscular mycorrhizae affect lowland tropical rain forest plant growth. - Ecology 61: 151-162. https://doi.org/10.2307/1937165

Jones, B. J. (2001): Laboratory guide for conducting soil and plant analysis. - CRC Press, Boca Raton, FL, 384 pp.

Jumpponen, A. and Trappe, J. M. (1998): Dark septate endophytes: a review of facultative biotrophic root colonizing fungi. - New Phytol. 140: 295-310. https://doi.org/10.1046/ j.1469-8137.1998.00265.x

Koske, R. E. and Gemma, J. N. (1989): A modified procedure for staining roots to detect VA mycorrhizae. - Mycol. Res. 92: 486-488. https://doi.org/10.1016/s0953-7562(09)80692-8

Koske, R. E. and Gemma, J. N. (1992): Fungal reactions to plants prior to mycorrhizal formation. - In: Allen, M. F. (ed.): Mycorrhizal functioning: an integrative plant fungal process. Chapman \& Hall, New York, pp. 3-27.

Kumar, S. and Adholeya, A. (2016): Impact of land use and soil types on arbuscular mycorrhizal fungal diversity in tropical soil of India. - Afr. J. Microbiol. Res. 10: 1595-1606. https://doi.org/10.5897/ajmr2016.8192

Lakshmipathy, R., Balakrishna, A. N. and Bagyaraj, D. J. (2012): Abundance and diversity of AM fungi across a gradient of land use intensity and their seasonal variations in Niligiri biosphere of the Western Ghats, India. - J. Agr. Sci. Tech. 14: 903-918.

Li, T., Li, J.-P. and Zhao, Z.-W. (2004): Arbuscular mycorrhizas in a valley-type savanna in a southwest China. - Mycorrhiza 14: 323-327. https://doi.org/10.1007/s00572-003-0277-y

Lovelock, C. E., Andersen, K. and Morton, J. B. (2003): Arbuscular mycorrhizal communities in tropical forests are affected by host tree species and environment. - Oecologia 135: 268-279. https://doi.org/10.1007/s00442-002-1166-3

Maherali, H. (2014): Is there an association between root architecture and mycorrhizal growth response? - New Phytol. 204: 192-200. https://doi.org/10.1111/nph.12927 
Mandyam, K. and Jumpponen, A. (2005): Seeking the elusive function of the root-colonising dark septate endophytic fungi. - Stud. Mycol. 53: 173-189. https://doi.org/10.3114/ sim.53.1.173

Mangan, S. A., Eom, A. H., Adler, G. H., Yavitt, J. B. and Herre, E. A. (2004): Diversity of arbuscular mycorrhizal fungi across a fragmented forest in Panama: insular spore communities differ from mainland communities. - Oecologia 141: 687-700. https:// doi.org/10.1007/s00442-004-1684-2

Matekwor Ahulu, E., Nakata, M. and Nonaka, M. (2005): Arum- and Paris-type arbuscular mycorrhizas in a mixed pine forest on sand dune soil in Niigata pretecture, central Honashu, Japan. - Mycorrhiza 15: 129-136. https://doi.org/10.1007/s00572-004-0310-9

McGonigle, T. P., Miller, M. H., Evans, D. G., Fairchild, G. L. and Swan, J. A. (1990): A new method which gives an objective measure of colonization of roots by vesicular-arbuscular mycorrhizal fungi. - New Phytol. 115: 495-501. https://doi. org/10.1111/j.1469-8137.1990.tb00476.x

McGuire, K. L., Henkel, T. W., de la Cerda, I. G., Villa, G., Edmund, F. and Andrew, C. (2008): Dual mycorrhizal colonization of forest-dominating tropical trees and the mycorrhizal status of non-dominant tree and liana species. - Mycorrhiza 18: 217-222. https://doi.org/10.1007/s00572-008-0170-9

Murugesan, M. (2005): Floristic diversity and ethnobotanical studies in Velliangiri hills, the Western Ghats of Coimbatore district, Tamil Nadu, India. - PhD thesis, Bharathiar University.

Murugesan, M. and Balasubramaniam, V. (2008): A survey on the orchids of Velliangiri hills, a part of Nilgiri Biosphere Reserve, India, with special reference to Indian endemics. - J. Sci. Trans. Environ. Techn. 1: 186-200. https://doi.org/10.20894/stet.116.001.004.004

Muthukumar, T. and Prabha, K. (2013): Arbuscular mycorrhizal and septate endophyte fungal associations in lycophytes and ferns of South India. - Symbiosis 59: 15-33. https://doi.org/10.1007/s13199-012-0185-z

Muthukumar, T. and Udaiyan, K. (2000a): Arbuscular mycorrhizas of plants growing in the Western Ghats region, southern India. - Mycorrhiza 9: 297-313. https://doi. org/10.1007/s005720050274

Muthukumar, T. and Udaiyan, K. (2000b): Vesicular arbuscular mycorrhizae in pteridophytes of Western Ghats, South India. - Phytomorphol. 50: 132-142.

Muthukumar, T. and Udaiyan, K. (2002): Arbuscular mycorrhizae in cycads of southern India. - Mycorrhiza 12: 213-217. https://doi.org/10.1007/s00572-002-0179-4

Muthukumar, T., Udaiyan, K. and Manian, S. (1996): Vesicular-arbuscular mycorrhizae in tropical sedges of southern India. - Biol. Fertil. Soils 22: 96-100. https://doi. org/10.1007/bf00384439

Muthukumar, T., Udaiyan, K. and Shanmughavel, P. (2004): Mycorrhiza in sedges - an overview. - Mycorrhiza 14: 65-77. https://doi.org/10.1007/s00572-004-0296-3

Muthukumar, T., Senthilkumar, M., Rajangam, M. and Udaiyan, K. (2006): Arbuscular mycorrhizal morphology and dark septate fungal association in medicinal and aromatic plants of Western Ghats, Southern India. - Mycorrhiza 17: 11-24. https:/doi. org/10.1007/s00572-006-0077-2

Muthukumar, T., Udaiyan, K., Karthikeyan, A. and Manian, S. (1997): Influence of native endomycorrhiza, soil flooding and nurse plant on mycorrhizal status and growth of purple nutsedge (Cyperus rotundus L.). - Agric. Ecosyst. Environ. 61: 51-58. https:// doi.org/10.1016/s0167-8809(96)01073-0 
Muthukumar, T., Sha, L. Q., Yang, X. D., Cao, M., Tang, J. W. and Zheng, Z. (2003): Mycorrhiza of plants in different vegetation types in tropical ecosystems of Xishuangbanna, southwest China. - Mycorrhiza 13: 289-297. https://doi.org/10.1007/s00572-003-0234-9

Nair, N. C. and Henry, A. N. (1983): Flora of Tamil Nadu, India. Vol. 1. - Botanical Survey of India, Coimbatore, India, $184 \mathrm{pp}$.

Newsham, K. K. (2011): A meta-analysis of plant responses to dark septate root endophytes. - New Phytol. 190: 783-793. https://doi.org/10.1111/j.1469-8137.2010.03611.x

Onguene, N. A. and Kuyper, T. W. (2001): Mycorrhizal associations in the rain forest of south Cameroon. - For. Ecol. Manage. 140: 277-287. https://doi.org/10.1016/s03781127(00)00322-4

Öpik, M. and Davison, J. (2016): Uniting species- and community-oriented approaches to understand arbuscular mycorrhizal fungal diversity. - Fungal Ecol. 24: 106-113. https://doi.org/10.1016/j.funeco.2016.07.005

Phillips, R. P. and Fahey, T. J. (2006): Tree species and mycorrhizal associations influence the magnitude of rhizosphere effects. - Ecology 87: 1302-1313. https://doi. org/10.1890/0012-9658(2006)87[1302:tsamai]2.0.co;2

Picone, C. (2000): Diversity and abundance of arbuscular-mycorrhizal fungal spores in tropical forest and pasture. - Biotropica 32: 734-747. https://doi.org/10.1646/00063606(2000)032[0734:daaoam]2.0.co;2

Prain, D. (1981a): Bengal Plants. Vol. 1. - Bishen Singh Mahendrapal Singh, Dehradun, India, $663 \mathrm{pp}$.

Prain, D. (1981b): Bengal Plants. Vol. 2. Bishen Singh Mahendrapal Singh, Dehradun, India, $574 \mathrm{pp}$.

Radhika, K. P. and Rodrigues, B. F. (2010): Arbuscular mycorrhizal fungal diversity in some commonly occurring medicinal plants of Western Ghats, Goa region. - J. For. Res. 21: 45-52. https://doi.org/10.1007/s11676-010-0007-1

Ragupathy, S. and Mahadevan, A. (1993): Distribution of vesicular arbuscular mycorrhizae in the plants and rhizosphere soil of the tropical plains, Tamil Nadu, India. - Mycorrhiza 3: 123-136. https://doi.org/10.1007/bf00208920

Ragupathy, S., Steven, N. G., Maruthakkutti, M., Velusamy, B. and Ul-Huda, M. M. (2008): Consensus of the 'Malasars' traditional aboriginal knowledge of medicinal plants in the Velliangiri holy hills, India. - J. Ethnobiol. Ethnomed. 4: 8. https://doi. org/10.1186/1746-4269-4-8

Rains, K. C., Nadkarni, N. M. and Bledsoe, C. S. (2003): Epiphytic and terrestrial mycorrhizas in a lower montane Costa Rican cloud forest. - Mycorrhiza 13: 257-264. https:// doi.org/10.1007/s00572-003-0224-y

Sathiyadash, K., Muthukumar, T. and Uma, E. (2010): Arbuscular mycorrhizal and dark septate endophyte fungal associations in south Indian grasses. - Symbiosis 52: 21-32. https://doi.org/10.1007/s13199-010-0096-9

Schappe, T., Albornoz, F. E., Turner, B. L., Neat, A., Condit, R. and Jones, F. A. (2017): The role of soil chemistry and plant neighbourhoods in structuring fungal communities in three Panamanian rainforests. - J. Ecol. 105: 569-579. https://doi.org/10.1111/13652745.12752

Schenck, N. C. and Perez, Y. (1990): Manual for the identification of VA mycorrhizal fungi. Synergistic, Gainesville, Fl, 286 pp.

Schmidt, S. K., Sobieniak-Wiseman, L. C., Kageyama, S. A., Halloy, S. R. P. and Schadt, C. W. (2008): Mycorrhizal and dark-septate fungi in plant roots above 4270 meters elevation in the Andes and rocky mountains. - Arct. Antarct. Alp. Res. 40: 576-583. https://doi.org/10.1657/1523-0430(07-068)[schmidt]2.0.co;2 
Schüßler, A. and Walker, C. (2010): The Glomeromycota. A species list with new families and new genera. - The Royal Botanic Garden Kew, Kew, England, 58 pp.

Smith, S. E. and Read, D. J. (2008): Mycorrhizal symbiosis. - Academic Press, Inc. San Diego California, 605 pp.

Smith, S. E., Jakobsen, I., Grønlund, M. and Smith, F. A. (2011): Roles of arbuscular mycorrhizas in plant phosphorus nutrition: Interactions between pathways of phosphorus uptake in arbuscular mycorrhizal roots have important implications for understanding and manipulating plant phosphorus acquisition. - Plant Physiol. 156: 1050-1057. https://doi.org/10.1104/pp.111.174581

St. John, T. V. and Koske, R. E. (1988): Statistical treatment of endogonaceous spore counts. - Trans. Br. Mycol. Soc. 91: 117-121. https://doi.org/10.1016/s0007-1536(88)80012-3

Stürmer, S. L. and Siqueira, J. O. (2006): Diversity of arbuscular mycorrhizal fungi in Brazilian ecosystems. - In: Moreira, F. M. S., Siqueira, J. O. and Brussaard, L. (eds): Soil biodiversity in Amazonian and other Brazilian ecosystems. Londres, pp. 206-236.

Suchitra, R., Kumutha, K. and Balachandar, D. (2012): Morpho-typing and molecular diversity of arbuscular mycorrhizal fungi in sub-tropical soils of Coimbatore region, Tamil Nadu, India. - Indian J. Microbiol. 52: 145-152. https://doi.org/10.1007/s12088011-0206-2

Tester, M., Smith, S. E. and Smith, F. A. (1987): The phenomenon of "nonmycorrhizal plants". - Can. J. Bot. 65: 419-431. https://doi.org/10.1139/b87-051

The Plant List (2013): Version 1.1. Published on the Internet; http://www.theplantlist.org/ (accessed 25th January 2017)

Toby, D. and Hodd, P. (1982): Grasses of Western India. - Bombay Natural History Society, Bombay, $168 \mathrm{pp}$.

Uma E., Muthukumar, T., Sathiyadash, K. and Muniappan, V. (2010): Mycorrhizal and dark septate fungal associations in gingers and spiral gingers. - Botany 88: 500-511. https://doi.org/10.1139/b10-021

Uma, E., Sathiyadash, K., Loganathan, J. and Muthukumar, T. (2012): Tree species as hosts for arbuscular mycorrhizal and dark septate endophyte fungi. - J. For. Res. 23: 641649. https://doi.org/10.1007/s11676-012-0267-z

Vandenkoornhuyse, P., Husband, R., Daniell, T. J., Watson, I. J., Duck, J. M., Fitter, A. H. and Young, J. P. W. (2002): Arbuscular mycorrhizal community composition associated with two plant species in a grassland ecosystem. - Mol. Ecol. 11: 1555-1564. https://doi.org/10.1046/j.1365-294x.2002.01538.x

Vierheilig, H., Bennett, R., Kiddle, G., Kaldorf, M. and Ludwig-Müller, J. (2000): Differences in glucosinolate patterns and arbuscular mycorrhizal status of glucosinolate containing species. - New Phytol. 146: 343-352. https://doi.org/10.1046/j.14698137.2000.00642.x

Wang, B. and Qiu, Y.-L. (2006): Phylogenetic distribution and evolution of mycorrhizas in land plants. - Mycorrhiza 16: 299-363. https://doi.org/10.1007/s00572-005-0033-6

Yamato, M. and Iwasaki, M. (2002): Morphological types and of arbuscular mycorrhizal fungi in roots of understory plants in Japanese deciduous broadleaved forests. $-\mathrm{My}$ corrhiza 12: 291-296. https://doi.org/10.1007/s00572-002-0187-4

Yang, G., Yang, X., Zhang, W., Wei, Y., Ge, G., Lu, W., Sun, J., Liu, N., Kan, H., Shen, Y. and Zhang, Y. (2016): Arbuscular mycorrhizal fungi affect plant community structure under various nutrient conditions and stabilize the community productivity. - Oikos 125: 576-585. https://doi.org/10.1111/oik.02351

Yang, W., Zheng, Y., Gao, C., He, X., Ding, Q., Kim, Y., Rui, Y., Wang, S. and Guoet, L.D. (2013): The arbuscular mycorrhizal fungal community response to warming and 
grazing differs between soil and roots on the Qinghai-Tibetan Plateau. - PLoS ONE 8: e76447. https://doi.org/10.1371/journal.pone.0076447.

Yu, J., Xue, Z., He, X., Liu, C. and Steinberger, Y. (2017): Shifts in composition and diversity of arbuscular mycorrhizal fungi and glomalin contents during revegetation of desertified semiarid grassland. - Appl. Soil Ecol. 115: 60-67. https://doi.org/10.1016/j. apsoil.2017.03.015

Zandavalli, R. B., Stürmer, S. L. and Dillenburg, L. R. (2008): Species richness of arbuscular mycorrhizal fungi in forest with Araucaria in southern Brazil. - Hoehnea 35: 63-68. https://doi.org/10.1590/s2236-89062008000100003

Zangaro, W., Nishidate, F. R., Vandresen, J., Andrade, G. and Nogueira, M. A. (2007): Root mycorrhizal colonization and plant responsiveness are related to root plasticity, soil fertility and successional status of native woody species in southern Brazil. - J. Trop. Ecol. 23: 53-62. https://doi.org/10.1017/s0266467406003713

Zangaro, W., Rostirola, L. V., de Souza, P. B., Alves, R. A., Lescano, L. E. A. M., Rondina, A. B. L., Nogueira, M. A. and Carrenho, R. (2013): Root colonization and spore abundance of arbuscular mycorrhizal fungi in distinct successional stages from an Atlantic rainforest biome in southern Brazil. - Mycorrhiza 23: 221-233. https://doi.org/10.1007/ s00572-012-0464-9

Zhang, T., Sun, Y., Shi, Z. and Feng, G. (2012): Arbuscular mycorrhizal fungi can accelerate the restoration of degraded spring grassland in Central Asia. - Range Ecol. Manage. 65: 426-432. https://doi.org/10.2111/rem-d-11-00016.1

Zhang, Y., Guo, L. D. and Liu, R. J. (2004): Survey of arbuscular mycorrhizal fungi in deforested and natural forest land in the subtropical region of Dujiangyan southwest China. - Plant Soil 261: 257-263. https://doi.org/10.1023/b:plso.0000035572.15098.f6

Zhao, Z. W., Wang, G. H. and Yang, L. (2003): Biodiversity of arbuscular mycorrhizal fungi in tropical rainforest of Xishuangbanna, southwest China. - Fungal Divers. 13: 233-242. 